\title{
Constructions for optimal Ferrers diagram rank-metric codes 1
}

\author{
Shuangqing Liu, Yanxun Chang, and Tao Feng \\ Department of Mathematics, Beijing Jiaotong University, Beijing 100044, P. R. China \\ 16118420@bjtu.edu.cn, yxchang@bjtu.edu.cn, tfeng@bjtu.edu.cn
}

\begin{abstract}
Optimal rank-metric codes in Ferrers diagrams can be used to construct good subspace codes. Such codes consist of matrices having zeros at certain fixed positions. This paper generalizes the known constructions for Ferrers diagram rank-metric (FDRM) codes. Via a criterion for linear maximum rank distance (MRD) codes, an explicit construction for a class of systematic MRD codes is presented, which is used to produce new optimal FDRM codes. By exploring subcodes of Gabidulin codes, if each of the rightmost $\delta-1$ columns in Ferrers diagram $\mathcal{F}$ has at least $n-r$ dots, where $r$ is taken in a range, then the conditions that an FDRM code in $\mathcal{F}$ is optimal are established. The known combining constructions for FDRM code are generalized by introducing the concept of proper combinations of Ferrers diagrams.
\end{abstract}

Keywords: Ferrers diagram, rank-metric code, Gabidulin code, subspace code.

\section{Introduction}

Network coding, introduced in [2, refers to coding at the intermediate nodes when information is multicasted in a network. Often information is modeled as vectors of fixed length over a finite field $\mathbb{F}_{q}$, called packets. To improve the performance of the communication, intermediate nodes should forward random linear $\mathbb{F}_{q \text {-combinations of }}$ the packets they receive. Hence, the vector space spanned by the packets injected at the source is globally preserved in the network when no error occurs.

This observation led Kötter and Kschischang [12] to model network codes as projective space $\mathcal{P}_{q}(n)$, the set of all subspaces of $\mathbb{F}_{q}^{n}$, or Grassmann space $\mathcal{G}_{q}(n, k)$, the set of all subspaces of $\mathbb{F}_{q}^{n}$ having dimension $k$. Subsets of $\mathcal{P}_{q}(n)$ are called subspace codes or projective codes, while subsets of the Grassmann space are referred to as constant-dimension codes or Grassmann codes. The subspace distance $d_{S}(U, V)=\operatorname{dim} U+\operatorname{dim} V-2 \operatorname{dim}(U \cap V)$ for all $U, V \in \mathcal{P}_{q}(n)$ is used as a distance measure for subspace codes. For more information on constructions and bounds for subspace codes, the interested reader may refer to 6 - 8, 10, 13, 17, 19, 21, 22,

Silva, Kschischang and Kötter [20] pointed out that lifted maximum rank distance (MRD) codes can result in almost optimal constant dimension codes, which asymptotically attain the known upper bounds [8,12], and can be decoded efficiently in the context of random linear network coding.

To obtain optimal constant dimension codes, Etzion and Silberstein [6] presented a simple but effective construction, named the multilevel construction, which generalizes the lifted MRD codes construction by introducing a new family of rank-metric codes, namely, Ferrers diagram rank-metric codes. Furthermore, Etzion, Gorla, Ravagnani

\footnotetext{
${ }^{1}$ Supported by NSFC under Grant 11431003, 11471032, and Fundamental Research Funds for the Central Universities under Grant 2016JBM071, 2016JBZ012
} 
and Wachter-Zeh [5] systematically investigated Ferrers diagram rank-metric codes and established four constructions to obtain optimal codes.

This paper continues the work in [5]. In Section 2, we give a brief introduction of Ferrers diagram rank-metric codes, and review most of known constructions in the literature.

Via a criterion for linear MRD codes presented in [23, we give an explicit construction for a class of systematic MRD codes in Section 3.1, which can be used to produce optimal Ferrers diagram rank-metric codes (see Construction 3.5). In Section 3.2, we generalize Construction 2 in [5] by exploring subcodes of Gabidulin codes. Construction 2 in [5] requires that each of the rightmost $\delta-1$ columns in Ferrers diagram $\mathcal{F}$ has at least $n-1$ dots. We relax the condition $n-1$ to $n-r$, where $r$ is taken in a range (see Theorem 3.13).

In Section 4, by introducing the concept of proper combinations of Ferrers diagrams, we generalize Theorem 9 in [5]. Our constructions are essentially to combine small Ferrers diagram rank-metric codes to a bigger one more flexibly (see Constructions 4.7, 4.10 and $4.13)$.

\section{Preliminaries}

Let $q$ be a prime power, $\mathbb{F}_{q}$ be the finite field of order $q$, and $\mathbb{F}_{q^{m}}$ be its extension field of order $q^{m}$. We use $\mathbb{F}_{q}^{m \times n}$ to denote the set of all $m \times n$ matrices over $\mathbb{F}_{q}$, and $\mathbb{F}_{q^{m}}^{n}$ to denote the set of all row vectors of length $n$ over $\mathbb{F}_{q^{m}}$. The rank of a matrix $\mathbf{A} \in \mathbb{F}_{q}^{m \times n}$ is denoted by $\operatorname{rank}(\mathbf{A})$. The rows and columns of an $m \times n$ matrix will be indexed by $0,1, \ldots, m-1$ and $0,1, \ldots, n-1$, respectively. Let $[n]$ denote $\{0,1, \ldots, n-1\}$ and $(i, j)$ denote the cell in the $i$-th row and the $j$-th column of an $m \times n$ matrix, where $i \in[n]$ and $j \in[m]$. Write $\mathbf{I}_{s}$ as the $s \times s$ identity matrix.

\subsection{Rank-metric codes}

The set $\mathbb{F}_{q}^{m \times n}$ is an $\mathbb{F}_{q}$-vector space. The rank distance on $\mathbb{F}_{q}^{m \times n}$ is defined by

$$
d_{R}(\mathbf{A}, \mathbf{B})=\operatorname{rank}(\mathbf{A}-\mathbf{B}) \text { for } \mathbf{A}, \mathbf{B} \in \mathbb{F}_{q}^{m \times n} .
$$

An $[m \times n, k, \delta]_{q}$ rank-metric code $\mathcal{C}$ is a $k$-dimensional $\mathbb{F}_{q}$-linear subspace of $\mathbb{F}_{q}^{m \times n}$ with minimum rank distance

$$
\delta=\min _{\mathbf{A}, \mathbf{B} \in \mathcal{C}, \mathbf{A} \neq \mathbf{B}}\left\{d_{R}(\mathbf{A}, \mathbf{B})\right\} .
$$

Clearly

$$
\delta=\min _{\mathbf{A} \in \mathcal{C}, \mathbf{A} \neq \mathbf{0}}\{\operatorname{rank}(\mathbf{A})\} .
$$

The Singleton-like upper bound for rank-metric codes implies that

$$
k \leq \max \{m, n\}(\min \{m, n\}-\delta+1)
$$

holds for any $[m \times n, k, \delta]_{q}$ code. When the equality holds, $\mathcal{C}$ is called a linear maximum rank distance code, denoted by an $\operatorname{MRD}[m \times n, \delta]_{q}$ code. Linear MRD codes exists for all feasible parameters (cf. [4, 9, 15]). 


\section{$2.2 \quad$ Ferrers diagram rank-metric codes}

Given positive integers $m$ and $n$, an $m \times n$ Ferrers diagram $\mathcal{F}$ is an $m \times n$ array of dots and empty cells such that all dots are shifted to the right of the diagram, the number of dots in each row is less than or equal to the number of dots in the previous row, and the first row has $n$ dots and the rightmost column has $m$ dots. The number of dots in $\mathcal{F}$ is denoted by $|\mathcal{F}|$.

\section{Example 2.1}

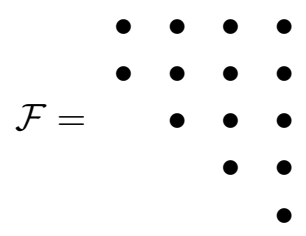

is a $5 \times 4$ Ferrers diagram and $|\mathcal{F}|=14$.

Sometimes it is convenient to state Ferrers diagrams by using the set-theoretical language (cf. [3,11]). Given positive integers $m$ and $n$, an $m \times n$ Ferrers diagram $\mathcal{F}$ is a subset of $[m] \times[n]$ satisfying that $(1)$ if $(i, j) \in \mathcal{F}$ and $i \geq 1$, then $(i-1, j) \in \mathcal{F}$; (2) if $(i, j) \in \mathcal{F}$ and $j \leq n-2$, then $(i, j+1) \in \mathcal{F}$. In the sequel, these two definitions will be both used, depending on what is more convenient in the context.

Motivated by the multilevel construction from [6], some research work has been done on constructing good or even optimal rank-metric codes in Ferrers diagrams [5, 11, 19,24. For a given $m \times n$ Ferrers diagram $\mathcal{F}$, an $[\mathcal{F}, k, \delta]_{q}$ Ferrers diagram rank-metric (FDRM) code, briefly an $[\mathcal{F}, k, \delta]_{q}$ code, is an $[m \times n, k, \delta]_{q}$ rank-metric code in which for each $m \times n$ matrix, all entries not in $\mathcal{F}$ are zero. If $\mathcal{F}$ is a full $m \times n$ diagram with $m n$ dots, then its corresponding FDRM code is just a classical rank-metric code.

Etzion and Silberstein [6] established a Singleton-like upper bound on FDRM codes.

Lemma 2.2 (Theorem 1 in [6]) Let $\delta$ be a positive integer. Let $\mathcal{F}$ be a Ferrers diagram and $\mathcal{C}_{\mathcal{F}}$ be any $[\mathcal{F}, k, \delta]_{q}$ code. Then $k \leq \min _{i \in[\delta]} v_{i}$, where $v_{i}$ is the number of dots in $\mathcal{F}$ which are not contained in the first $i$ rows and the rightmost $\delta-1-i$ columns.

An FDRM code which attains the upper bound in Lemma 2.2 is called optimal. An $\operatorname{MRD}[m \times n, \delta]_{q}$ code with $m \geq n$ is an optimal $[\mathcal{F}, m(n-\delta+1), \delta]_{q}$ code, where $\mathcal{F}$ is a full $m \times n$ diagram. So far all known FDRM codes over $\mathbb{F}_{q}$ with the largest possible dimension are optimal.

We remark that the upper bound still holds for FDRM codes defined on any field, and especially, for algebraically closed fields the bound cannot be attained (see Theorem 13 and Proposition 17 in [1]). This paper focuses only on finite fields since they are used for forming subspace codes.

For a Ferrers diagram $\mathcal{F}$ of size $m \times n$, one can transpose it to obtain a Ferrers diagram $\mathcal{F}^{t}$ of size $n \times m$. Thus if there exists an $[\mathcal{F}, k, \delta]_{q}$ code, then so does an $\left[\mathcal{F}^{t}, k, \delta\right]_{q}$ code. Without loss of generality, we always assume that $m \geq n$.

We denote by $\gamma_{i}, i \in[n]$, the number of dots in the $i$-th column of $\mathcal{F}$, and by $\rho_{i}$, $i \in[m]$, the number of dots in the $i$-th row of $\mathcal{F}$. 


\subsection{Known constructions for FDRM codes}

This section summarizes known main constructions for FDRM codes, which come from $[1,5,6,11,25]$. We shall use or generalize them later.

\subsubsection{Exploration of subcodes of MRD codes}

Etzion and Silberstein [6] introduced the concept of FDRM codes. They established the existence of optimal $[\mathcal{F}, k, \delta]_{q}$ codes whenever $\mathcal{F}$ is an $m \times n(m \geq n)$ Ferrers diagram and each of its rightmost $\delta-1$ columns has at least $m$ dots. The proof is based on the use of $q$-cyclic MRD codes. A better result is provided in [5] with a simple proof by means of shortening systematic MRD codes (see also Theorem 23 in [11] and Corollary 3.3 in [1]).

Theorem 2.3 (Theorem 3 in [5]) Assume $\mathcal{F}$ is an $m \times n$ Ferrers diagram and each of the rightmost $\delta-1$ columns of $\mathcal{F}$ has at least $n$ dots. Then there exists an optimal $[\mathcal{F}, k, \delta]_{q}$ code for any prime power $q$, where $k=\sum_{i=0}^{n-\delta} \gamma_{i}$.

As a straightforward corollary, Etzion and Silberstein pointed out the following fact.

Corollary 2.4 [6] Let $\delta \in\{1,2\}$. There exists an optimal $[\mathcal{F}, k, \delta]_{q}$ code for any Ferrers diagram $\mathcal{F}$ and any prime power $q$, where $k=\sum_{i=0}^{n-\delta} \gamma_{i}$.

To relax the restriction on $\mathcal{F}$ in Theorem 2.3, the idea of exploring subcodes of MRD codes was introduced to construct FDRM codes in [5], and developed in [1,25] recently.

Theorem 2.5 (Theorem 8 in [5]) Assume $\mathcal{F}$ is an $m \times n$ Ferrers diagram and $m \geq n$. Let $2 \leq \delta \leq n-1$. If each of the rightmost $\delta-1$ columns in $\mathcal{F}$ has at least $n-1$ dots, then there exists an $[\mathcal{F}, k, \delta]_{q}$ code for any prime power $q$, where $k=\min \left\{m-n+1, \gamma_{0}\right\}+\sum_{i=1}^{n-\delta} \gamma_{i}$. When $\gamma_{0} \leq m-n+1$, the resulting FDRM code is optimal.

We shall generalize Theorem 2.5 to Theorem 3.13, where it is required that each of the rightmost $\delta-1$ columns in $\mathcal{F}$ has at least $n-r$ dots for any positive integer $r$ satisfying $r+1 \leq \delta \leq n-r$.

Theorem 2.6 (Theorem 3.6 in [1]) Assume $\mathcal{F}$ is an $m \times n$ Ferrers diagram and $m \geq n$. Let $2 \leq \delta \leq n$ and $l=n-\delta+1$. Set $\varepsilon=\sum_{t=l}^{n-1}\left(m-\gamma_{t}\right)$, that is, $\varepsilon$ is the number of dots missing in the rightmost $\delta-1$ columns of $\mathcal{F}$. If $\gamma_{s} \leq \gamma_{l}-\varepsilon(l-s)$ for every $s \in\{0,1, \ldots, l-1\}$, then there exists an optimal $\left[\mathcal{F}, \sum_{i=0}^{l-1} \gamma_{i}, \delta\right]_{q}$ code.

Theorem 2.6 implies Theorem 2.3 when $\varepsilon=0$. When $\varepsilon \neq 0$, the condition $\gamma_{s} \leq$ $\gamma_{l}-\varepsilon(l-s)$ for $s \in\{0,1, \ldots, l-1\}$ means that the numbers of dots in the first $l$ columns are restricted in an arithmetic progression with step size $\varepsilon$.

Theorem 2.7 (Theorem 3.6 in [25]) Let $l$ be a positive integer. Let $1=t_{0}<t_{1}<t_{2}<$ $\cdots<t_{l}$ be integers such that $t_{1}\left|t_{2}\right| \cdots \mid t_{l}$. Let $n$ and $\delta$ be positive integers satisfying $t_{l-1}<n-1 \leq t_{l}$ and $n-t_{1}+1<\delta \leq n-1$. Let $\mathcal{F}$ be an $m \times n$ Ferrers diagram satisfying

(1) $\gamma_{n-\delta} \leq t_{1}$ 
(2) $\gamma_{n-\delta+1} \geq t_{1}$

(3) $\gamma_{t_{i}} \geq t_{i+1}$ for $1 \leq i \leq l-1$,

(4) $\gamma_{n-1} \geq t_{l}+\gamma_{0}$,

Then there exists an optimal $\left[\mathcal{F}, \sum_{i=0}^{n-\delta} \gamma_{i}, \delta\right]_{q}$ code for any prime power $q$.

When $l=1$, Theorem 2.7 together with Theorem 2.3 yields Theorem 2.5 (note that to remove the condition $\gamma_{n-\delta} \leq t_{1}$, Theorem 2.3 is needed).

\subsubsection{Use of MDS codes}

A construction for FDRM codes based on maximum distance separable (MDS) codes is presented in [5]. It is known that an $[n, n-d+1, d]_{q}$ MDS code exists for any $q \geq n-1$ or $d \in\{1,2, n\}$ (see [14]).

A diagonal of a Ferrers diagram $\mathcal{F}$ is a consecutive sequence of entries, going upwards diagonally from the rightmost column to either the leftmost column or the first row. Let $D_{i}, i \in[m]$, denote the $i$-th diagonal in $\mathcal{F}$, where $i$ counts the diagonals from the top to the bottom and let $\theta_{i}$ denote the number of dots on $D_{i}$ in $\mathcal{F}$.

Example 2.8 For the Ferrers diagram in Example 2.1, its five diagonals are:

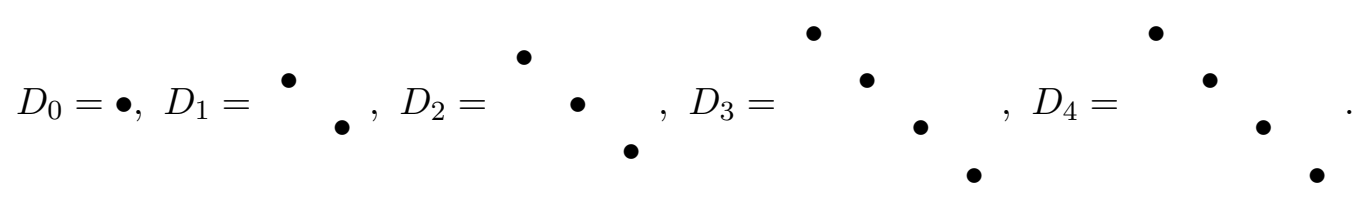

Theorem 2.9 (Construction 1 in $[5]$ ) Let $\mathcal{F}$ be an $m \times n$ Ferrers diagram and $\delta$ be an integer such that $0<\delta \leq n$. Let $\theta_{\max }=\max _{i \in[m]} \theta_{i}$. Then there exists an $[\mathcal{F}, k, \delta]_{q}$ code for any prime power $q \geq \theta_{\max }-1$, where $k=\sum_{i=0}^{m-1} \max \left\{0, \theta_{i}-\delta+1\right\}$.

Applying Theorems 2.3 and 2.9, Etzion and Silberstein obtained the following result.

Corollary 2.10 (Theorem 11 in [5]) Let $n \geq 3$. There exists an optimal $[\mathcal{F}, k, 3]_{q}$ code for any $n \times n$ Ferrers diagram $\mathcal{F}$ and any prime power $q$.

The disadvantage of Theorem 2.9 is the requirement of large $q$. For example when $\mathcal{F}$ is an $n \times n$ Ferrers diagram with $i+1$ dots in its $i$-th column for $i \in[n]$, by Theorem 2.9, there exists an optimal $[\mathcal{F}, 3, n-1]_{q}$ code for any prime power $q \geq n-1$. Recently Antrobus and Gluesing-Luerssen showed that such optimal FDRM codes exist for any prime power $q$ via induction on $n$.

Theorem 2.11 (Theorem 5.2 in [1]) Let $n \geq 3$. Assume $\mathcal{F}$ is an $n \times n$ Ferrers diagram with $i+1$ dots in its $i$-th column for $i \in[n]$. Then there exists an optimal $[\mathcal{F}, 3, n-1]_{q}$ code for any prime power $q$.

However, how to give other constructions for FDRM codes with the same parameters as those obtained from Theorem 2.9, but for any prime power $q$, is still an open problem. We shall exhibit three examples in Section 3 (Examples [3.6, 3.8, 3.9) to touch this problem. 


\subsubsection{Combination of FDRM codes}

To obtain new FDRM codes based on known ones, [5] presented an excellent idea. We shall extend this idea in Section 4.

Theorem 2.12 (Theorem 9 in [5]) Let $\mathcal{F}_{i}$ for $i=1,2$ be an $m_{i} \times n_{i}$ Ferrers diagram, and $\mathcal{C}_{i}$ be an $\left[\mathcal{F}_{i}, k, \delta_{i}\right]_{q}$ code. Let $\mathcal{D}$ be an $m_{3} \times n_{3}$ full Ferrers diagram with $m_{3} n_{3}$ dots, where $m_{3} \geq m_{1}$ and $n_{3} \geq n_{2}$. Let

$$
\mathcal{F}=\left(\begin{array}{cc}
\mathcal{F}_{1} & \mathcal{D} \\
& \mathcal{F}_{2}
\end{array}\right)
$$

be an $m \times n$ Ferrers diagram, where $m=m_{2}+m_{3}$ and $n=n_{1}+n_{3}$. Then there exists an $\left[\mathcal{F}, k, \delta_{1}+\delta_{2}\right]_{q}$ code.

The limitation of Theorem 2.12 can be shown in the following lemma.

Lemma 2.13 Let $\delta$ be a positive integer. Let $\mathcal{F}$ be an $m \times n$ Ferrers diagram satisfying

$$
\max _{n-\delta+1 \leq i \leq n-1}\left(\gamma_{i}-\gamma_{i-1}\right)<v_{0}=\min _{i \in[\delta]} v_{i}
$$

where $v_{i}$ is the number of dots in $\mathcal{F}$ which are not contained in the first $i$ rows and the rightmost $\delta-1-i$ columns. Then one cannot apply Theorem 2.12 to construct an optimal $\left[\mathcal{F}, v_{0}, \delta\right]_{q}$ code.

Proof Assume that an optimal $\left[\mathcal{F}, v_{0}, \delta\right]_{q}$ code can be constructed by Theorem 2.12 , where

$$
\mathcal{F}=\left(\begin{array}{cc}
\mathcal{F}_{1} & \mathcal{D} \\
& \mathcal{F}_{2}
\end{array}\right)
$$

is an $m \times n$ Ferrers diagram, $\mathcal{F}_{j}$ is an $m_{j} \times n_{j}$ Ferrers diagram, $\mathcal{C}_{j}$ is an $\left[\mathcal{F}_{j}, v_{0}, \delta_{j}\right]_{q}$ code for $j=1,2$, and $\delta=\delta_{1}+\delta_{2}$. Let $v_{0}^{(j)}$ be the number of dots in $\mathcal{F}_{j}$ which are not contained in the rightmost $\delta_{j}-1$ columns.

Consider $\mathcal{C}_{1}$. By Lemma 2.2, $v_{0} \leq v_{0}^{(1)}$, i.e., $\sum_{i=0}^{n-\delta} \gamma_{i} \leq \sum_{i=0}^{n_{1}-\delta_{1}} \gamma_{i}$, which yields $n-\delta \leq n_{1}-\delta_{1}$. Thus $n_{1} \geq n-\delta+\delta_{1}$ and $\delta_{2}=\delta-\delta_{1} \geq n-n_{1}$.

Consider $\mathcal{C}_{2}$. Since $\delta_{2} \leq n_{2} \leq n-n_{1}$, we have $\delta_{2}=n_{2}=n-n_{1}$. By Lemma 2.2. the existence of an $\left[\mathcal{F}_{2}, v_{0}, n_{2}\right]_{q}$ code implies $v_{0}$ is no more than the number of dots in $\mathcal{F}_{2}$ which are not contained in the rightmost $n_{2}-1$ columns. Hence, $v_{0} \leq \gamma_{n_{1}}-\gamma_{n_{1}-1}$, which contradicts with the known condition $\max _{n-\delta+1 \leq i \leq n-1}\left(\gamma_{i}-\gamma_{i-1}\right)<v_{0}$.

\section{Constructions based on subcodes of MRD codes}

Let $\boldsymbol{\beta}=\left(\beta_{0}, \beta_{1}, \ldots, \beta_{m-1}\right)$ be an ordered basis of $\mathbb{F}_{q^{m}}$ over $\mathbb{F}_{q}$. There is a natural bijective map $\Psi_{m}$ from $\mathbb{F}_{q^{m}}^{n}$ to $\mathbb{F}_{q}^{m \times n}$ as follows:

$$
\begin{gathered}
\Psi_{m}: \mathbb{F}_{q^{m}}^{n} \longrightarrow \mathbb{F}_{q}^{m \times n} \\
\mathbf{a}=\left(a_{0}, a_{1}, \ldots, a_{n-1}\right) \longmapsto \mathbf{A},
\end{gathered}
$$


where $\mathbf{A}=\Psi_{m}(\mathbf{a}) \in \mathbb{F}_{q}^{m \times n}$ is defined such that

$$
a_{j}=\sum_{i=0}^{m-1} A_{i, j} \beta_{i}
$$

for any $j \in[n]$. For $a \in \mathbb{F}_{q^{m}},(a)$ is a $1 \times 1$ matrix and we simply write $\Psi_{m}((a))$ as $\Psi_{m}(a)$. It is readily checked that $\Psi_{m}$ satisfies linearity, i.e., $\Psi_{m}\left(x \mathbf{c}_{1}+y \mathbf{c}_{2}\right)=x \Psi_{m}\left(\mathbf{c}_{1}\right)+y \Psi_{m}\left(\mathbf{c}_{2}\right)$ for any $x, y \in \mathbb{F}_{q}$ and $\mathbf{c}_{1}, \mathbf{c}_{2} \in \mathbb{F}_{q^{m}}^{n}$. The map $\Psi_{m}$ will be used to facilitate switching between a vector in $\mathbb{F}_{q^{m}}$ and its matrix representation over $\mathbb{F}_{q}$. In the sequel, we use both representations, depending on what is more convenient in the context and by slight abuse of notation, $\operatorname{rank}(\mathbf{a})$ denotes $\operatorname{rank}\left(\Psi_{m}(\mathbf{a})\right)$.

The following lemma, implicitly shown in Section 5 in [5], is fundamental to construct FDRM codes via subcodes of MRD codes. All theorems in Section 2.3.1 are based on this lemma.

Lemma 3.1 [5] Assume that $m \geq n$. Let $\boldsymbol{G}$ be a generator matrix of a systematic $M R D[m \times n, \delta]_{q}$ code, i.e., $\boldsymbol{G}$ is of the form $\left(\boldsymbol{I}_{k} \mid \boldsymbol{A}\right)$, where $k=n-\delta+1$. Let $0 \leq \lambda_{0} \leq \lambda_{1} \leq \cdots \leq \lambda_{k-1} \leq m$. Let $\boldsymbol{U}=\left\{\left(u_{0}, \ldots, u_{k-1}\right) \in \mathbb{F}_{q^{m}}^{k}: \Psi_{m}\left(u_{i}\right)=\right.$ $\left.\left(u_{i, 0}, \ldots, u_{i, \lambda_{i}-1}, 0, \ldots, 0\right)^{T}, u_{i, j} \in \mathbb{F}_{q}, i \in[k], j \in\left[\lambda_{i}\right]\right\}$. Then $\mathcal{C}=\left\{\Psi_{m}(\boldsymbol{c}): \boldsymbol{c}=\boldsymbol{u} \boldsymbol{G}, \boldsymbol{u} \in\right.$ $\boldsymbol{U}\}$ is a linear FDRM code with dimension $\sum_{i=0}^{k-1} \lambda_{i}$ and rank at least $\delta$ over $\mathbb{F}_{q}$.

Proof One can easily verify the linearity and the dimension of the code. Since $\mathbf{G}$ is a generator matrix of an $\operatorname{MRD}[m \times n, \delta]_{q}$ code $\mathcal{C}_{M}, \mathcal{C}$ is a subcode of $\mathcal{C}_{M}$. So the minimum rank distance of the code $\mathcal{C}$ is $\delta$.

Lemma 3.1 doesn't show the Ferrers diagram used explicitly. However, if we could know more about the initial MRD code, then it would be possible to give a complete characterization of $\mathcal{C}$.

Remark 3.2 Lemma 3.1 can only be used to construct optimal FDRM codes satisfying $v_{0}=\sum_{i=0}^{n-\delta} \gamma_{i}=\min _{i \in[\delta]} v_{i}$ (cf. Lemma 2.2), where $v_{i}$ is the number of dots in $\mathcal{F}$ which are not contained in the first $i$ rows and the rightmost $\delta-1-i$ columns.

\subsection{Construction from a class of systematic MRD codes}

To construct systematic MRD codes, we need the following theorem, which provides a criterion for linear MRD codes.

Theorem 3.3 23] Let $m \geq n$. Let $\boldsymbol{G} \in \mathbb{F}_{q^{m}}^{k \times n}$ be a generator matrix of a linear rankmetric code $\mathcal{C} \subseteq \mathbb{F}_{q^{m}}^{n}$. Then $\mathcal{C}$ is an $M R D$ code if and only if for any $\boldsymbol{B} \in \operatorname{UT}_{n}^{*}(q)$ every maximal minor of $\boldsymbol{G B}$ is nonzero, where $U T_{n}^{*}(q)$ denotes the set of all $n \times n$ upper triangular matrices over $\mathbb{F}_{q}$ whose main diagonal elements are all 1.

Lemma 3.4 Let $q$ be a prime power. Let $m, n$ and $\delta$ be positive integers satisfying $m>n \geq \delta \geq 2$. Let $k=n-\delta+1$ and $m \geq k n-k^{2}+2$. Let $\left(1, \beta, \beta^{2}, \ldots, \beta^{m-1}\right)$ be an ordered polynomial basis of $\mathbb{F}_{q^{m}}$ over $\mathbb{F}_{q}$. If there exists a $k \times n$ matrix

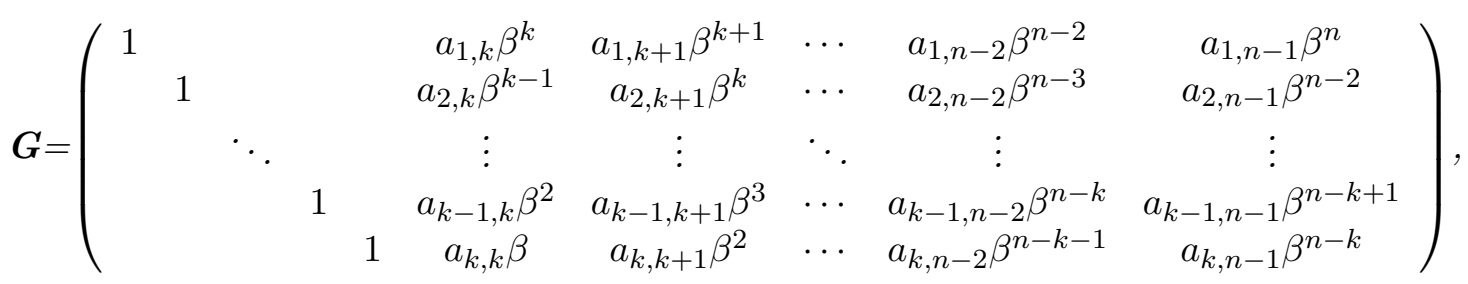


where $a_{i, j} \in \mathbb{F}_{q}^{*}, i \in\{1,2, \ldots, k\}$ and $j \in\{k, k+1, \ldots, n-1\}$, such that every minor of the matrices

$$
\boldsymbol{A}_{1}=\left(\begin{array}{ccc}
a_{1, k} & \cdots & a_{1, n-2} \\
\vdots & \ddots & \vdots \\
a_{k, k} & \cdots & a_{k, n-2}
\end{array}\right) \text { and } \boldsymbol{A}_{2}=\left(\begin{array}{ccc}
a_{2, k} & \cdots & a_{2, n-1} \\
\vdots & \ddots & \vdots \\
a_{k, k} & \cdots & a_{k, n-1}
\end{array}\right)
$$

is nonzero, then $\boldsymbol{G}$ is a generator matrix of a systematic $M R D[m \times n, \delta]_{q}$ code.

Proof Obviously, $n-k \geq 0$. By Theorem 3.3, it suffices to prove that for any $\mathbf{B} \in U T_{n}^{*}(q)$, every $k$-minor of $\mathbf{G B}$ is nonzero. To ensure smooth reading of the paper, we move the proof to Appendix $\mathrm{A}$.

For a vector $\left(v_{1}, v_{2}, \ldots, v_{n}\right)$ of length $n$, if its rightmost nonzero component is $v_{r}$ for some $1 \leq r \leq n$, then $r$ is said to be the valid length of this vector.

Construction 3.5 Let $m, n$ and $\delta$ be positive integers satisfying $m>n \geq \delta \geq 2$. Let $k=n-\delta+1$ and $m \geq k n-k^{2}+2$. If there exists a $k \times n$ matrix $\boldsymbol{G}$ satisfying the condition in Lemma 3.4 such that $\boldsymbol{G}$ is a generator matrix of a systematic $M R D[m \times n, \delta]_{q}$ code, then there exists an optimal $\left[\mathcal{F}, \sum_{i=0}^{k-1} \gamma_{i}, \delta\right]_{q}$ code $\mathcal{C}$ for any $m^{\prime} \times n$ Ferrers diagram $\mathcal{F}$ satisfying

(1) $\gamma_{i}=\min \left\{\max \left\{\gamma_{l}+i-l: l \in[k]\right\}, m\right\}$ for any $k \leq i \leq n-2$,

(2) $m^{\prime}=\min \left\{\max \left\{\gamma_{0}+n, \max \left\{\gamma_{l}+n-1-l: 1 \leq l \leq k-1\right\}\right\}, m\right\}$,

where $\gamma_{i}, i \in[n]$, is the number of dots in the $i$-th column of $\mathcal{F}$.

Proof Start from the generator matrix $\mathbf{G}$ of the given systematic MRD code. We can apply Lemma 3.1 by setting $\lambda_{i}=\gamma_{i}, i \in[k]$, to obtain an optimal FDRM code $\mathcal{C}$ in some Ferrers diagram $\mathcal{F}$ with dimension $\sum_{i=0}^{k-1} \gamma_{i}$ and rank at least $\delta$. It suffices to analyze the number of dots in each column of $\mathcal{F}$.

By Lemma 3.1, for any $\mathbf{c}=\left(c_{0}, c_{1}, \ldots, c_{n-1}\right) \in \mathcal{C}$, we have $\mathbf{c}=\mathbf{u G}$ for some $\mathbf{u}=$ $\left(u_{0}, u_{1}, \ldots, u_{k-1}\right)$.

When $0 \leq i \leq k-1, c_{i}=u_{i}$, and so $\Psi_{m}\left(c_{i}\right)=\Psi_{m}\left(u_{i}\right)=\left(u_{i, 0}, \ldots, u_{i, \gamma_{i}-1}, 0, \ldots, 0\right)$. Thus, the $i$-th column of $\mathcal{F}$ has $\gamma_{i}$ dots.

When $k \leq i \leq n-2, c_{i}=\sum_{l=0}^{k-1} u_{l} a_{l+1, i} \beta^{i-l}$ and so $\Psi_{m}\left(c_{i}\right)=\sum_{l=0}^{k-1} a_{l+1, i} \Psi_{m}\left(u_{l} \beta^{i-l}\right)$. For $l \in[k], \Psi_{m}\left(u_{l}\right)=\left(u_{l, 0}, u_{l, 1}, \ldots, u_{l, \gamma_{l}-1}, 0, \ldots, 0\right)^{T}$ implies $u_{l}=u_{l, 0}+u_{l, 1} \beta+\cdots+$ $u_{l, \gamma_{l}-1} \beta^{\gamma_{l}-1}$. Note that $\beta^{m+j}$ can be written as a linear combination of $1, \beta, \beta^{2}, \cdots, \beta^{m-1}$ for any nonnegative integer $j$. It follows that for each $l \in[k]$, as a vector of length $m$, $\Psi_{m}\left(u_{l} \beta^{i-l}\right)$ has a valid length of at most $\min \left\{\gamma_{l}+i-l, m\right\}$. Thus $\Psi_{m}\left(c_{i}\right)$ has a valid length of at most $\max \left\{\gamma_{l}+i-l: l \in[k]\right\}$ if $\max \left\{\gamma_{l}+i-l: l \in[k]\right\} \leq m$, or $m$ otherwise, which coincides with Condition (1).

When $i=n-1, c_{n-1}=\sum_{l=1}^{k-1} u_{l} a_{l+1, n-1} \beta^{n-1-l}+u_{0} a_{1, n-1} \beta^{n}$ and so $\Psi_{m}\left(c_{n-1}\right)=$ $\sum_{l=1}^{k-1} a_{l+1, n-1} \Psi_{m}\left(u_{l} \beta^{n-1-l}\right)+a_{1, n-1} \Psi_{m}\left(u_{0} \beta^{n}\right)$. As a vector of length $m, \Psi_{m}\left(u_{0} \beta^{n}\right)$ has a valid length of at most $\min \left\{\gamma_{0}+n, m\right\}$. For each $1 \leq l \leq k-1, \Psi_{m}\left(u_{l} \beta^{n-1-l}\right)$ has a valid length of at most $\min \left\{\gamma_{l}+n-1-l, m\right\}$. Thus $\Psi_{m}\left(c_{i}\right)$ has a valid length of at most $\max \left\{\gamma_{0}+n, \gamma_{1}+n-1-1, \gamma_{2}+n-1-2, \ldots, \gamma_{k-1}+n-1-(k-1)\right\}$ if $\max \left\{\gamma_{0}+n, \gamma_{1}+n-1-1, \gamma_{2}+n-1-2, \ldots, \gamma_{k-1}+n-1-(k-1)\right\} \leq m$, or $m$ otherwise, which coincides with Condition (2). 
Example 3.6 Let $q$ be a prime power. Let $m \geq 2 n-2$ and $3 \leq n \leq q+2$. Let $\left(1, \beta, \ldots, \beta^{m-1}\right)$ be an ordered polynomial basis of $\mathbb{F}_{q^{m}}$ over $\mathbb{F}_{q}$. Construct a $2 \times n$ matrix

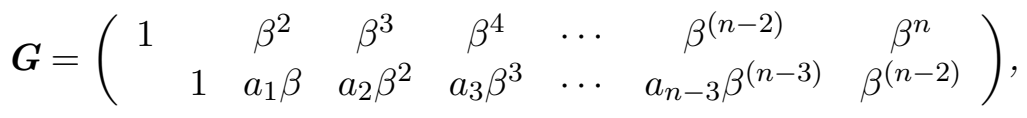

where $a_{i} \in \mathbb{F}_{q}^{*}, 1 \leq i \leq n-3$, and $a_{i} \neq a_{j}$ for any $i \neq j$. By Lemma 3.4, $\boldsymbol{G}$ is a generator matrix of a systematic $M R D[m \times n, n-1]_{q}$ code.

Let $\mathcal{F}$ be an $m^{\prime} \times n$ Ferrers diagram satisfying $\gamma_{0} \leq m-n, \gamma_{i}=\gamma_{0}+i$ for $1 \leq i \leq n-2$ and $\gamma_{n-1}=m^{\prime}=\gamma_{0}+n$. It is readily checked that $\mathcal{F}$ satisfies Conditions (1) and (2) in Construction 3.5. Thus there exists an optimal $\left[\mathcal{F}, \gamma_{0}+\gamma_{1}, n-1\right]_{q}$ code.

Remark 3.7 When $q=n-2$, Example 3.6 cannot be obtained from Theorem 2.9 since no $[n, 2, n-1]_{n-2} M D S$ code exists, even though here Theorem 2.9 can deal with all cases of prime power $q \geq n-1$. No known construction can be applied here to handle the case of $q=n-2$. It is readily checked that Theorems 2.3,2.6 are invalid. Consider Theorem 2.7 with $l \geq 2$ (when $l=1$, Theorem 2.7 degenerates into Theorem 2.3). Condition (1)

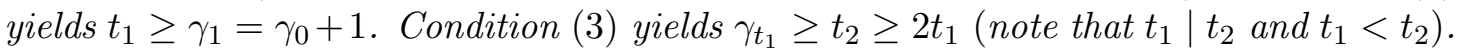
Since $\gamma_{t_{1}}=\gamma_{0}+t_{1}$, we have $t_{1} \leq \gamma_{0}$, a contradiction. Thus Theorem 2.7 is also invalid. When $\gamma_{0}>1$, Example 3.6 cannot be obtained from Theorem 2.11. By using Lemma 2.13 (note that $\max _{n-\delta+1 \leq i \leq n-1}\left(\gamma_{i}-\gamma_{i-1}\right)=2$ and $v_{0}=\gamma_{0}+\gamma_{1}=2 \gamma_{0}+1$ ), we have that Theorem 2.12 is invalid. Similar arguments hold for the following two examples.

Example 3.8 Let $q=5, n=7$ and $m \geq 14$. Let $\left(1, \beta, \ldots, \beta^{m-1}\right)$ be an ordered polynomial basis of $\mathbb{F}_{5^{m}}$ over $\mathbb{F}_{5}$. Construct a $3 \times 7$ matrix

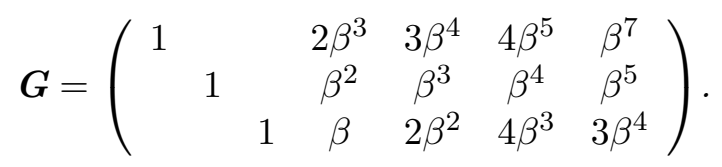

By Lemma 3.4, $\boldsymbol{G}$ is a generator matrix of a systematic $M R D[m \times 7,5]_{5}$ code.

Let $\mathcal{F}$ be an $m^{\prime} \times n$ Ferrers diagram satisfying $\gamma_{0} \leq m-7, \gamma_{i}=\gamma_{0}+i$ for $1 \leq i \leq 5$ and $\gamma_{6}=m^{\prime}=\gamma_{0}+7$. It is readily checked that $\mathcal{F}$ satisfies Conditions (1) and (2) in Construction 3.5. Thus there exists an optimal $\left[\mathcal{F}, \gamma_{0}+\gamma_{1}+\gamma_{2}, 5\right]_{5}$ code.

Example 3.9 Let $q=7, n=9$ and $m \geq 20$. Let $\left(1, \beta, \ldots, \beta^{m-1}\right)$ be an ordered polynomial basis of $\mathbb{F}_{7^{m}}$ over $\mathbb{F}_{7}$. Construct a $3 \times 9$ matrix

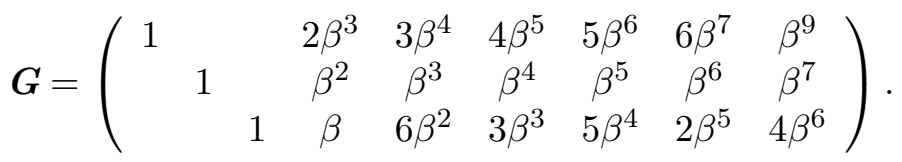

By Lemma 3.4, $\boldsymbol{G}$ is a generator matrix of a systematic $M R D[m \times 9,7]_{7}$ code.

Let $\mathcal{F}$ be an $m^{\prime} \times n$ Ferrers diagram satisfying $\gamma_{0} \leq m-9, \gamma_{i}=\gamma_{0}+i$ for $1 \leq i \leq 7$ and $\gamma_{8}=m^{\prime}=\gamma_{0}+9$. It is readily checked that $\mathcal{F}$ satisfies Conditions (1) and (2) in Construction 3.5. Thus there exists an optimal $\left[\mathcal{F}, \gamma_{0}+\gamma_{1}+\gamma_{2}, 7\right]_{7}$ code.

Remark 3.10 In Lemma 3.4, the top right entry of $\boldsymbol{G}$ uses the $n$-th power of $\beta$, which deviates from the patter in the rest of $\boldsymbol{G}$. If $a_{1, n-1} \beta^{n-1}$ is taken as the top right entry in $\boldsymbol{G}$, then similar arguments to those in the proof of Lemma 3.4 show that $\boldsymbol{G}$ is a generator matrix of a systematic $M R D[m \times n, \delta]_{q}$ code if every minor of the matrix 


$$
\left(\begin{array}{ccccc}
a_{1, k} & a_{1, k+1} & \cdots & a_{1, n-2} & a_{1, n-1} \\
a_{2, k} & a_{2, k+1} & \cdots & a_{2, n-2} & a_{2, n-1} \\
\vdots & \vdots & \ddots & \vdots & \vdots \\
a_{k, k} & a_{k, k+1} & \cdots & a_{k, n-2} & a_{k, n-1}
\end{array}\right)
$$

is nonzreo. However, so far we have not found any appropriate $\boldsymbol{G}$ such that new optimal FDRM codes can be derived from it.

\subsection{Construction based on subcodes of Gabidulin codes}

For any positive integer $i$ and any $a \in \mathbb{F}_{q^{m}}$, set $a^{[i]} \triangleq a^{q^{i}}$. In this section, we shall generalize Construction 2 in [5] by exploring subcodes of Gabidulin codes.

Let $m \geq n$ and $q$ be any prime power. A Gabidulin code $\mathcal{G}[m \times n, \delta]_{q}$ is an $\operatorname{MRD}[m \times$ $n, \delta]_{q}$ code whose generator matrix $\mathbf{G}$ in vector representation is

$$
\mathbf{G}=\left(\begin{array}{cccc}
g_{0} & g_{1} & \cdots & g_{n-1} \\
g_{0}^{[1]} & g_{1}^{[1]} & \cdots & g_{n-1}^{[1]} \\
\vdots & \vdots & \ddots & \vdots \\
g_{0}^{[n-\delta]} & g_{1}^{[n-\delta]} & \cdots & g_{n-1}^{[n-\delta]}
\end{array}\right)
$$

where $g_{0}, g_{1}, \ldots, g_{n-1} \in \mathbb{F}_{q^{m}}$ are linearly independent over $\mathbb{F}_{q}$ (see [9]).

The following lemma is a generalization of Lemma 5 in [5], which only deals with the case of $r=1$. We move its proof to Appendix B.

Lemma 3.11 Let $\eta, r, d, \kappa$ and $\mu$ be positive integers such that $\kappa=\eta-r-d+1, r<\kappa$ and $\eta \leq \mu+r$. Then there exists a matrix $\boldsymbol{G} \in \mathbb{F}_{q^{\mu}}^{\kappa \times \eta}$ of the following form

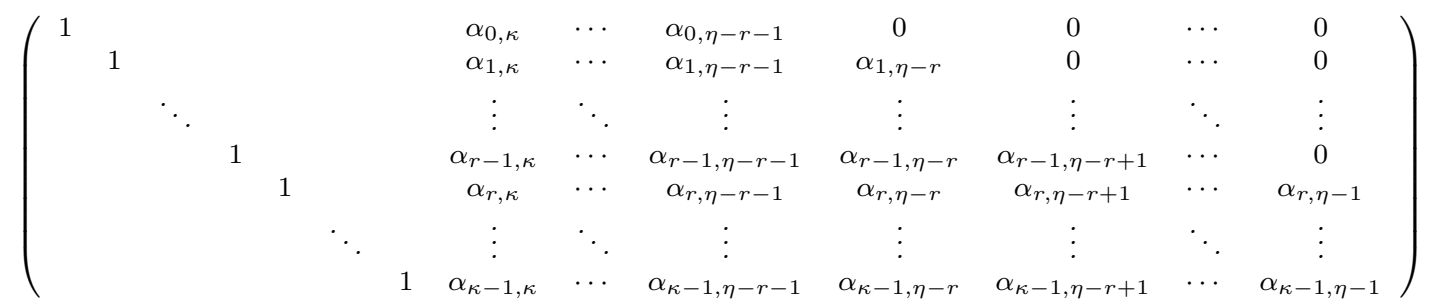

satisfying that for each $0 \leq i \leq r$, the sub-matrix obtained by removing the first $i$ rows, the leftmost $i$ columns and the rightmost $r-i$ columns of $\boldsymbol{G}$ can produce an $M R D[\mu \times$ $(\eta-r), d+i]_{q}$ code.

Construction 3.12 Let $\delta, n$ and $r$ be positive integers satisfying $r+1 \leq \delta \leq n-r$. To take a $\kappa \times \eta$ matrix $\boldsymbol{G}$ satisfying Lemma 3.11, assume that $d=\delta-r, \kappa=n-\delta+1$, $\eta=n$ and $\mu=n-r$. Let $\mathcal{F}$ be an $m \times n$ Ferrers diagram, each of whose rightmost $\delta-1$ columns has at least $n-r$ dots. Let $\gamma_{i}, i \in[n]$, is the number of dots in the $i$-th column of $\mathcal{F}$. For $l \in[r]$, set $s_{l}=\min \left\{\gamma_{l}-1, \gamma_{n-r+l}-n+r-\sum_{j=0}^{l-1}\left(s_{j}+1\right)-1\right\}$. Let

$$
\begin{gathered}
\boldsymbol{U}=\left\{\left(u_{0}, \ldots, u_{\kappa-1}\right) \in \mathbb{F}_{q^{n-r}}^{\kappa}: \Psi_{n-r}\left(u_{l}\right)=\left(u_{l, 0}, \ldots, u_{l, s_{l}}, 0, \ldots, 0\right)^{T} \text { for } l \in[r],\right. \\
\left.\Psi_{n-r}\left(u_{l}\right)=\left(u_{l, 0}, \ldots, u_{l, \gamma_{l}-1}, 0, \ldots, 0\right)^{T} \text { for } r \leq l \leq \kappa-1, \text { all possible } u_{i, j} \in \mathbb{F}_{q}\right\} . \\
\text { Let } \bar{\Psi}_{n-r}\left(u_{l}\right)=\left(u_{l, 0}, \ldots, u_{l, s_{l}}\right)^{T} \text { for } l \in[r] . \text { If } 0 \leq s_{0} \leq s_{1} \leq \cdots \leq s_{r-1}, \text { then } \mathcal{C}=
\end{gathered}
$$




$$
\left\{\left(\begin{array}{ccccccc}
\multicolumn{7}{c}{\Psi_{n-r}(\boldsymbol{c})} \\
\hline 0 & \cdots & 0 & \bar{\Psi}_{n-r}\left(u_{0}\right) & \bar{\Psi}_{n-r}\left(u_{1}\right) & \cdots & \bar{\Psi}_{n-r}\left(u_{r-1}\right) \\
0 & \cdots & 0 & 0 & \bar{\Psi}_{n-r}\left(u_{0}\right) & \ddots & \bar{\Psi}_{n-r}\left(u_{r-2}\right) \\
\vdots & & \vdots & \vdots & \vdots & \ddots & \vdots \\
0 & \cdots & 0 & 0 & 0 & \cdots & \bar{\Psi}_{n-r}\left(u_{0}\right)
\end{array}\right) \in \mathbb{F}_{q}^{m \times n}: \boldsymbol{c}=\boldsymbol{u} \cdot \boldsymbol{G}, \boldsymbol{u} \in \boldsymbol{U}\right\}
$$

is an $[\mathcal{F}, k, \delta]_{q}$ code for any prime power $q$, where $k=\sum_{i=0}^{r-1} s_{i}+r+\sum_{i=r}^{n-\delta} \gamma_{i}$. Note that $m=n+\sum_{i=0}^{r-1} s_{i}$.

Proof One can easily verify the linearity and the dimension of the code. It remains to examine the minimum rank weight of any nonzero codeword $\mathbf{C}$ from $\mathcal{C}$. Note that $\delta \leq n-r$, so $r \leq n-\delta<n-\delta+1=\kappa$.

Let $\mathbf{C}$ be formed by $\mathbf{c}=\mathbf{u G}=\left(u_{0}, u_{1}, \ldots, u_{\kappa-1}\right) \mathbf{G}$. Let $i^{*}=\min \left\{i: i \in[\kappa], u_{i} \neq\right.$ $0, u_{j}=0$ for any $\left.j<i\right\}$. Then $\mathbf{c}=\left(0, \ldots, 0, u_{i^{*}}, \ldots, u_{\kappa-1}\right) \mathbf{G}$.

(1) If $i^{*}<r$, then let $\Psi_{n-r}^{*}(\mathbf{u G})$ be an $(n-r) \times(n-r)$ matrix obtained by removing the leftmost $i^{*}$ columns and the rightmost $r-i^{*}$ columns of $\Psi_{n-r}(\mathbf{u G})$. By Lemma 3.11, $\Psi_{n-r}^{*}(\mathbf{u G})$ is a codeword of an $\operatorname{MRD}\left[\mu \times(n-r), \delta-r+i^{*}\right]_{q}$ code, whose generator matrix can be obtained by removing the first $i^{*}$ rows, the leftmost $i^{*}$ columns and the rightmost $r-i^{*}$ columns of $\mathbf{G}$. Thus $\operatorname{rank}\left(\Psi_{n-r}^{*}(\mathbf{u G})\right) \geq \delta-r+i^{*}$.

Furthermore, under the broken line of $\mathbf{C}$, since $\bar{\Psi}_{n-r}\left(u_{i^{*}}\right)$ with the length $s_{i^{*}}$ is a nonzero vector, the rightmost $r-i^{*}$ columns contribute rank $r-i^{*}$. Therefore, $\operatorname{rank}(\mathbf{C}) \geq$ $\operatorname{rank}\left(\Psi_{n-r}^{*}(\mathbf{u G})\right)+r-i^{*} \geq \delta-r+i^{*}+r-i^{*}=\delta$.

(2) If $i^{*} \geq r$, then let $\Psi_{n-r}^{*}(\mathbf{u G})$ be an $(n-r) \times(n-r)$ matrix obtained by removing the leftmost $r$ columns of $\Psi_{n-r}(\mathbf{u G})$. By Lemma 3.11, $\Psi_{n-r}^{*}(\mathbf{u G})$ is a codeword of an $\operatorname{MRD}[\mu \times(n-r), \delta]_{q}$ code, whose generator matrix can be obtained by removing the first $r$ rows and the leftmost $r$ columns of $\mathbf{G}$. Thus $\operatorname{rank}(\mathbf{C}) \geq \delta$.

Theorem 3.13 Let $\delta, n$ and $r$ be positive integers satisfying $r+1 \leq \delta \leq n-r$. Let $\mathcal{F}$ be an $m \times n$ Ferrers diagram satisfying that

(1) $\gamma_{n-\delta} \leq n-r$,

(2) $\gamma_{n-\delta+1} \geq n-r$

(3) $\gamma_{n-r+l} \geq n-r+\sum_{j=0}^{l} \gamma_{j}$ for $l \in[r]$.

Then there exists an optimal $\left[\mathcal{F}, \sum_{i=0}^{n-\delta} \gamma_{i}, \delta\right]_{q}$ code for any prime power $q$.

Proof Note that $r+1 \leq \delta$ yields $n-\delta \leq n-r-1$. Consider a new Ferrers diagram $\mathcal{F}^{\prime}$ with $\gamma_{i}^{\prime}$ for $i \in[n]$ as the number of dots in its $i$-th column, satisfying

$$
\gamma_{i}^{\prime}=\left\{\begin{array}{l}
\gamma_{i}, \text { if } 0 \leq i \leq n-\delta \\
n-r, \text { if } n-\delta+1 \leq i \leq n-r-1 \\
n-r+\sum_{j=0}^{i+r-n} \gamma_{j}, \quad \text { if } n-r \leq i \leq n-1 .
\end{array}\right.
$$

Then $\mathcal{F}^{\prime}$ is a sub-Ferrers diagram of $\mathcal{F}$ and $\gamma_{i}^{\prime} \leq n-r$ for $0 \leq i \leq n-r-1$. According to Lemma 2.2, it suffices to show that there exists an optimal $\left[\mathcal{F}^{\prime}, \sum_{i=0}^{n-\delta} \gamma_{i}^{\prime}, \delta\right]_{q}$ code, which implies the existence of an optimal $\left[\mathcal{F}, \sum_{i=0}^{n-\delta} \gamma_{i}, \delta\right]_{q}$ code. 
Clearly, each of the rightmost $\delta-1$ columns of $\mathcal{F}^{\prime}$ has at least $n-r$ dots. To apply Construction [3.12, we need to count $s_{l}$ for $l \in[r]$. By Condition (3), $s_{0}=$ $\min \left\{\gamma_{0}^{\prime}-1, \gamma_{n-r}^{\prime}-n+r-1\right\}=\gamma_{0}^{\prime}-1$. It follows that by induction on $l, l \in[r]$, we have $s_{l}=\min \left\{\gamma_{l}^{\prime}-1, \gamma_{n-r+l}^{\prime}-n+r-\sum_{j=0}^{l-1}\left(s_{j}+1\right)-1\right\}=\min \left\{\gamma_{l}^{\prime}-1, \gamma_{n-r+l}^{\prime}-\right.$ $\left.n+r-\sum_{j=0}^{l-1} \gamma_{j}^{\prime}-1\right\}=\gamma_{l}^{\prime}-1$. Thus $0 \leq s_{0} \leq s_{1} \leq \cdots \leq s_{r-1}$, and Construction 3.12 provides an $\left[\mathcal{F}^{\prime}, \sum_{i=0}^{n-\delta} \gamma_{i}^{\prime}, \delta\right]_{q}$ code, which is optimal by Lemma 2.2.

We remark that as a corollary of Theorem 3.13 with $r=1$, we can obtain Theorem 8 in [5].

Example 3.14 Let $n \geq 3$ and

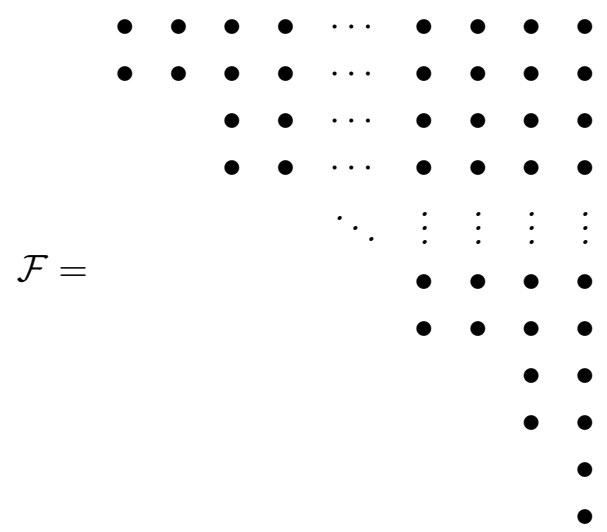

be a $(2 n+2) \times 2 n$ Ferrers diagram. Take $\delta=4$. Apply Theorem 3.13 with $r=2$. One can check that the rightmost 3 columns of $\mathcal{F}$ have at least $2 n-2$ dots, $\gamma_{2 n-2}=2 n=2 n-2+\gamma_{0}$ and $\gamma_{2 n-1}=2 n+2=2 n-2+\gamma_{0}+\gamma_{1}$. So $\mathcal{F}$ satisfies the conditions of Theorem 3.13 , and an optimal $\left[\mathcal{F}, 2(n-1)^{2}, 4\right]_{q}$ code exists for any prime power $q$.

Remark 3.15 No known construction can be applied to obtain Example 3.14. It is readily checked that Theorems $2.3,2.6$ and 2.11 are invalid. Consider Theorem 2.7 with $l \geq 2$. Condition (1) yields $t_{1} \geq \gamma_{2 n-4}=2 n-2$ and Condition (3) yields $\gamma_{t_{1}} \geq t_{2} \geq$ $2 t_{1} \geq 4 n-4$. Due to $\gamma_{t_{1}} \leq 2 n+2$, we have $n \leq 3$. So Theorem 2.7 is invalid when $n>3$. Actually by more careful calculation, one can check that Theorem 2.7 is also invalid for $n=3$. We leave the details to the interested reader. Theorem 2.9 can provide an $\left[\mathcal{F}, 2 n^{2}-4 n, 4\right]_{q}$ code for any prime power $q \geq 2 n-1$, but it is not optimal. By using Lemma 2.13 (note that $\max _{n-\delta+1 \leq i \leq n-1}\left(\gamma_{i}-\gamma_{i-1}\right)=2$ and $\left.v_{0}=2(n-1)^{2}\right)$, one can see that Theorem 2.12 is invalid.

\section{New Ferrers diagram rank-metric codes from old}

First we give a slight variation of Theorem 2.12, It is not complicated, but inspires us to establish this section.

Theorem 4.1 Let $\mathcal{F}_{i}$ for $i=1,2$ be an $m_{i} \times n_{i}$ Ferrers diagram, and $\mathcal{C}_{i}$ be an $\left[\mathcal{F}_{i}, k_{i}, \delta_{i}\right]_{q}$ code. Let $\mathcal{D}$ be an $m_{3} \times n_{3}$ Ferrers diagram and $\mathcal{C}_{3}$ be a $\left[\mathcal{D}, k_{3}, \delta\right]_{q}$ code, where $m_{3} \geq m_{1}$ and $n_{3} \geq n_{2}$. Let $m=m_{2}+m_{3}$ and $n=n_{1}+n_{3}$. Let

$$
\mathcal{F}=\left(\begin{array}{cc}
\mathcal{F}_{1} & \hat{\mathcal{D}} \\
& \mathcal{F}_{2}
\end{array}\right)
$$


be an $m \times n$ Ferrers diagram $\mathcal{F}$, where $\hat{\mathcal{D}}$ is obtained by adding the fewest number of new dots to the lower-left corner of $\mathcal{D}$ such that $\mathcal{F}$ is a Ferrers diagram. Then there exists an $\left[\mathcal{F}, \min \left\{k_{1}, k_{2}\right\}+k_{3}, \min \left\{\delta_{1}+\delta_{2}, \delta\right\}\right]_{q}$ code $\mathcal{C}$ satisfying that for any codeword $\boldsymbol{C} \in \mathcal{C}$, $\left.\boldsymbol{C}\right|_{\mathcal{F}_{1}}=\boldsymbol{O}$ if and only if $\left.\boldsymbol{C}\right|_{\mathcal{F}_{2}}=\boldsymbol{O}$, where $\left.\boldsymbol{C}\right|_{\mathcal{F}_{i}}$ denotes the restriction of $\boldsymbol{C}$ in $\mathcal{F}_{i}$ for $i=1,2$.

Proof Obviously, $\mathcal{C}_{3}$ is also a $\left[\hat{\mathcal{D}}, k_{2}, \delta\right]_{q}$ code, and the existence of $\left[\mathcal{F}_{i}, k_{i}, \delta_{i}\right]_{q}$ code $\mathcal{C}_{i}$ for $i=1,2$ implies the existence of $\left[\mathcal{F}_{i}, \min \left\{k_{1}, k_{2}\right\}, \delta_{i}\right]_{q}$ code $\mathcal{C}_{i}^{\prime}$. Let $\varphi$ be an isomorphism $\varphi: \mathcal{C}_{1}^{\prime} \longrightarrow \mathcal{C}_{2}^{\prime}$ (in the sense of linear spaces) and set

$$
\mathcal{C}=\left\{\left(\begin{array}{cc}
\mathbf{X} & \mathbf{D} \\
\mathbf{0} & \varphi(\mathbf{X})
\end{array}\right): \mathbf{X} \in \mathcal{C}_{1}^{\prime}, \mathbf{D} \in \mathcal{C}_{3}\right\}
$$

Clearly $\mathcal{C}$ is a linear code of dimension $\min \left\{k_{1}, k_{2}\right\}+k_{3}$. It suffices to examine the minimum rank distance of $\mathcal{C}$. Take any nonzero codeword $\mathbf{C}$ from $\mathcal{C}$. Since $\mathbf{X}$ and $\varphi(\mathbf{X})$ are either both zero or both nonzero, we consider the following two cases:

$\bullet \mathbf{X} \neq \mathbf{0}$.

$$
\operatorname{rank}(\mathbf{C})=\operatorname{rank}\left(\begin{array}{cc}
\mathbf{X} & \mathbf{D} \\
\mathbf{0} & \varphi(\mathbf{X})
\end{array}\right) \geq \operatorname{rank}(\mathbf{X})+\operatorname{rank}(\varphi(\mathbf{X})) \geq \delta_{1}+\delta_{2} .
$$

$\bullet \mathbf{X}=\mathbf{0}$.

$$
\operatorname{rank}(\mathbf{C})=\operatorname{rank}(\mathbf{D}) \geq \delta .
$$

Therefore, $\mathcal{C}$ is an $\left[\mathcal{F}, \min \left\{k_{1}, k_{2}\right\}+k_{3}, \min \left\{\delta_{1}+\delta_{2}, \delta\right\}\right]_{q}$ code.

\subsection{Generalization of Theorem 4.1}

To obtain optimal FDRM codes, in the process of using Theorem 4.1, it is often required that $\mathcal{C}_{3}$ is an optimal $\left[\mathcal{D}, k_{2}, \delta\right]_{q}$ code. If the optimality of $\mathcal{C}_{3}$ is unknown, then what shall we do? A natural idea is to remove a sub-diagram from $\mathcal{D}$ to obtain a new Ferrers diagram $\mathcal{D}^{\prime}$ such that the FDRM code in $\mathcal{D}^{\prime}$ is optimal, and then mix the removed subdiagram to $\mathcal{F}_{1}$ or $\mathcal{F}_{2}$. We shall illustrate the idea by using Example 4.5. Note that for this example, one can check that any known constructions cannot attain the required dimension.

First, we introduce a new concept. Let $\mathcal{F}_{1}$ be an $m_{1} \times n_{1}$ Ferrers diagram, $\mathcal{F}_{2}$ be an $m_{2} \times n_{2}$ Ferrers diagram and $\mathcal{F}$ be an $m \times n$ Ferrers diagram. Let $\phi_{l}$ for $l \in\{1,2\}$ be an injection from $\mathcal{F}_{l}$ to $\mathcal{F}$ (in the sense of set-theoretical language). $\mathcal{F}$ is said to be a proper combination of $\mathcal{F}_{1}$ and $\mathcal{F}_{2}$ on a pair of mappings $\phi_{1}$ and $\phi_{2}$, if

(1) $\phi_{1}\left(\mathcal{F}_{1}\right) \cap \phi_{2}\left(\mathcal{F}_{2}\right)=\varnothing$;

(2) $\left|\mathcal{F}_{1}\right|+\left|\mathcal{F}_{2}\right|=|\mathcal{F}|$;

(3) for any $l \in\{1,2\}$ and any two different elements $\left(i_{l, 1}, j_{l, 1}\right),\left(i_{l, 2}, j_{l, 2}\right)$ of $\mathcal{F}_{l}$, set $\phi_{l}\left(i_{l, 1}, j_{l, 1}\right)=\left(i_{l, 1}^{\prime}, j_{l, 1}^{\prime}\right)$ and $\phi_{l}\left(i_{l, 2}, j_{l, 2}\right)=\left(i_{l, 2}^{\prime}, j_{l, 2}^{\prime}\right) ; i_{l, 1}^{\prime}=i_{l, 2}^{\prime}$ or $j_{l, 1}^{\prime}=j_{l, 2}^{\prime}$ whenever $i_{l, 1}=i_{l, 2}$ or $j_{l, 1}=j_{l, 2}$.

Condition (3) means that if two dots in $\mathcal{F}_{l}$ for $l \in\{1,2\}$ are in the same row or same column, then their corresponding two dots in $\mathcal{F}$ are also in the same row or same column.

Example 4.2 Let 


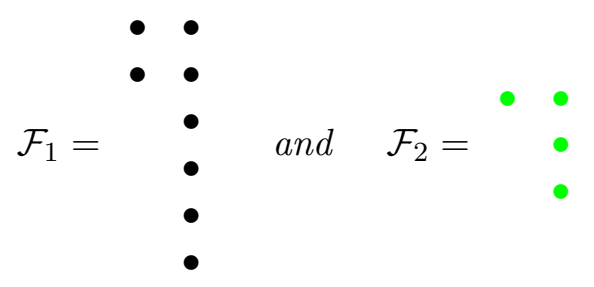

be two Ferrers diagrams. Then all of

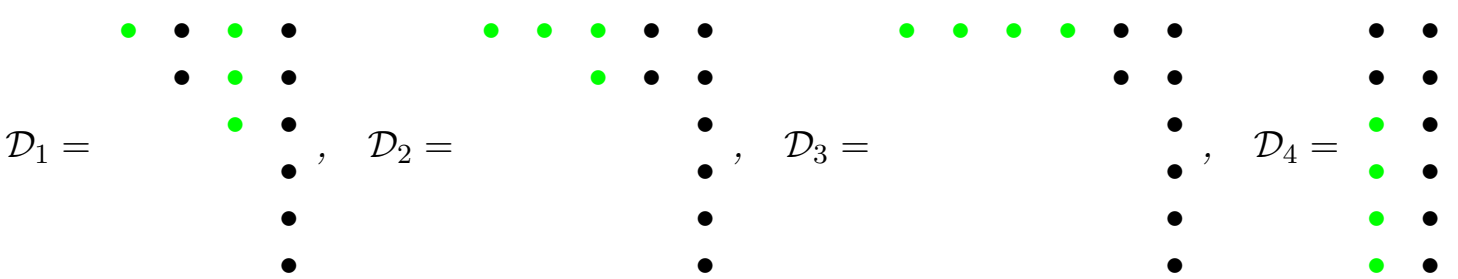

are proper combinations of $\mathcal{F}_{1}$ and $\mathcal{F}_{2}$. Note that $\mathcal{F}_{1}$ keeps its shape invariant in $\mathcal{D}_{l}$ for any $l \in\{1,2,3,4\} ; \mathcal{F}_{2}$ keeps its shape invariant in $\mathcal{D}_{1}$ and $\mathcal{D}_{2}$ (the transpose of $\mathcal{F}_{2}$ is allowed); $\mathcal{F}_{2}$ degenerates into a single row or column in $\mathcal{D}_{3}$ and $\mathcal{D}_{4}$.

Proposition 4.3 Let $\mathcal{F}$ be a proper combination of Ferrers diagrams $\mathcal{F}_{1}$ and $\mathcal{F}_{2}$. Then for each $l \in\{1,2\}$, either $\mathcal{F}_{l}$ keeps its shape invariant in $\mathcal{F}$ (the transpose of $\mathcal{F}_{l}$ is allowed), or $\mathcal{F}_{l}$ degenerates into a single row or column in $\mathcal{F}$.

Proof For $l \in\{1,2\}$, if $\mathcal{F}_{l}$ only contains one row or one column, then the conclusion follows immediately. Assume that $R_{1}$ and $R_{2}$ are two different rows of $\mathcal{F}_{l}$, and $R_{1}$ contains at least two dots. It is readily checked that these two rows either keep their shape invariant in $\mathcal{F}$ (a transpose is allowed), or degenerate into a single row $R$ or column $C$ in $\mathcal{F}$. If it is the latter, then by considering the rightmost two dots of $R_{1}$ and $R_{2}$ in $\mathcal{F}_{l}$ we have that the rightmost column in $\mathcal{F}_{l}$ must degenerate into the row $R$ or column $C$ in $\mathcal{F}$ (we refer to it as Fact A), and for any dot $P$ in any row (if this row exists) of $\mathcal{F}_{l}$ other than $R_{1}$ and $R_{2}$, if $P$ is not in the rightmost column, then there exists one dot $P^{\prime}$ in the rightmost column of $\mathcal{F}_{l}$ such that $P$ and $P^{\prime}$ are in the same row, which yields that $P$ and $P^{\prime}$ must degenerate into the same row or column in $\mathcal{F}$. Now it suffices to show that the row must be $R$ or the column must be $C$.

Since $R_{1}$ contains at least two dots, there exists a $\operatorname{dot} P^{\prime \prime}$ in $\mathcal{F}_{l}$ such that (i) $P^{\prime \prime}$ is not in the rightmost column, (ii) $P^{\prime \prime}$ and $P\left(P^{\prime \prime}\right.$ could be $P$ ) are in the same row, and (iii) there exists a $\operatorname{dot} P^{\prime \prime \prime}$ in $R_{1}$ such that $P^{\prime \prime}$ and $P^{\prime \prime \prime}$ are in the same column. Because of Fact A, $P^{\prime}$ and $P^{\prime \prime \prime}$ degenerate into the row $R$ or the column $C$ in $\mathcal{F}$. This forces $P$, $P^{\prime}, P^{\prime \prime}$ and $P^{\prime \prime \prime}$ to degenerate into the row $R$ or column $C$. Therefore, all dots in $\mathcal{F}_{l}$ must degenerate into the single row $R$ or column $C$ in $\mathcal{F}$.

Lemma 4.4 For $l \in\{1,2\}$, let $\mathcal{F}_{l}$ be an $m_{l} \times n_{l}$ Ferrers diagram and $\boldsymbol{M}_{l}$ be an $m_{l} \times n_{l}$ matrix whose entries not in $F_{l}$ are all zero. Let $\mathcal{F}$ be a proper combination of $\mathcal{F}_{1}$ and $\mathcal{F}_{2}$ on a pair of mappings $\phi_{1}$ and $\phi_{2}$ such that $\mathcal{F}$ is an $m \times n$ Ferrers diagram. Let $\boldsymbol{M}_{12}$ be an $m \times n$ matrix satisfying

$$
\boldsymbol{M}_{12}(i, j)=\left\{\begin{array}{l}
\boldsymbol{M}_{1}\left(i_{1}, j_{1}\right), \quad \text { if }(i, j)=\phi_{1}\left(i_{1}, j_{1}\right) \text { and }\left(i_{1}, j_{1}\right) \in \mathcal{F}_{1} \\
\boldsymbol{M}_{2}\left(i_{2}, j_{2}\right), \text { if }(i, j)=\phi_{2}\left(i_{2}, j_{2}\right) \text { and }\left(i_{2}, j_{2}\right) \in \mathcal{F}_{2} \\
0, \text { otherwise. }
\end{array}\right.
$$

Then $\operatorname{rank}\left(\boldsymbol{M}_{12}\right) \leq \operatorname{rank}\left(\boldsymbol{M}_{1}\right)+\operatorname{rank}\left(\boldsymbol{M}_{2}\right)$. 
Proof For $l \in\{1,2\}$, denote by $\left.\boldsymbol{M}_{12}\right|_{\mathcal{F}_{l}}$ the restriction of $\boldsymbol{M}_{12}$ in $\mathcal{F}_{l}$, i.e., $\left.\boldsymbol{M}_{12}\right|_{\mathcal{F}_{l}}$ is an $m \times n$ matrix satisfying

$$
\left.\boldsymbol{M}_{12}\right|_{\mathcal{F}_{l}}(i, j)=\left\{\begin{array}{l}
\boldsymbol{M}_{12}(i, j), \text { if }(i, j) \in \phi_{l}\left(\mathcal{F}_{l}\right) ; \\
0, \text { otherwise. }
\end{array}\right.
$$

Then using the basic fact that $\operatorname{rank}(\boldsymbol{A}+\boldsymbol{B}) \leq \operatorname{rank}(\boldsymbol{A})+\operatorname{rank}(\boldsymbol{B})$, we have $\operatorname{rank}\left(\boldsymbol{M}_{12}\right) \leq$ $\operatorname{rank}\left(\left.\boldsymbol{M}_{12}\right|_{\mathcal{F}_{1}}\right)+\operatorname{rank}\left(\boldsymbol{M}_{12} \mid \mathcal{F}_{2}\right)$. By Proposition $4.3, \operatorname{rank}\left(\left.\boldsymbol{M}_{12}\right|_{\mathcal{F}_{l}}\right) \leq \operatorname{rank}\left(\boldsymbol{M}_{l}\right)$ for $l \in$ $\{1,2\}$. The conclusion is then straightforward.

Example 4.5 We here construct an optimal $[\mathcal{F}, 10,4]_{q}$ code $\mathcal{C}$ for any prime power $q$, where

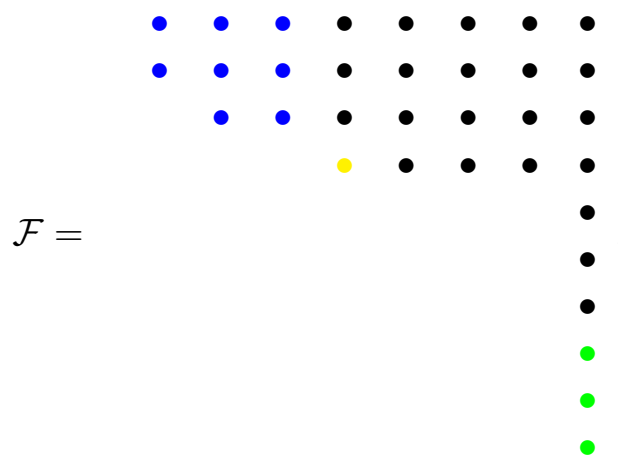

First take the following four Ferrers sub-diagrams of $\mathcal{F}$ :

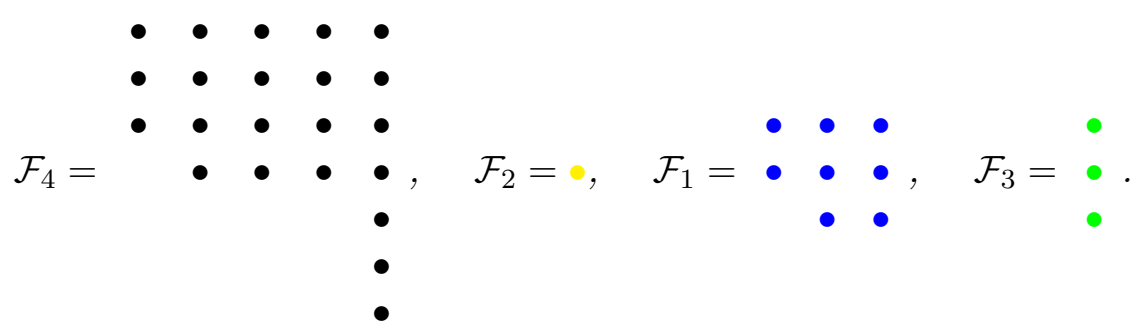

Then take a proper combination $\mathcal{F}_{12}$ of $\mathcal{F}_{1}$ and $\mathcal{F}_{2}$ on mappings $\phi_{1}$ and $\phi_{2}$ as follows

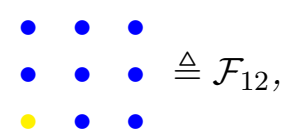

where $\phi_{1}: \mathcal{F}_{1} \longrightarrow \mathcal{F}_{12}$ satisfies $\phi_{1}(i, j)=(i, j)$ for any $(i, j) \in([3] \times[3]) \backslash\{(2,0)\}$, and $\phi_{2}: \mathcal{F}_{2} \longrightarrow \mathcal{F}_{12}$ satisfies $\phi_{2}(0,0)=(2,0)$. Now construct a new Ferrers diagram

$$
\mathcal{F}^{*}=\left(\begin{array}{cc}
\mathcal{F}_{12} & \mathcal{F}_{4} \\
& \mathcal{F}_{3}
\end{array}\right)
$$

For any prime power $q$, by Theorem 4.1 , we have an $\left[\mathcal{F}^{*}, 10,4\right]_{q}$ code $\mathcal{C}^{*}$ satisfying that for any $\boldsymbol{D} \in \mathcal{C}^{*},\left.\boldsymbol{D}\right|_{\mathcal{F}_{12}}=\boldsymbol{O}$ if and only if $\left.\boldsymbol{D}\right|_{\mathcal{F}_{3}}=\boldsymbol{O}$, where an optimal $\left[\mathcal{F}_{12}, 3,3\right]_{q}$ code $\mathcal{C}_{12}$ exists by Theorem 2.3 , an optimal $\left[\mathcal{F}_{4}, 7,4\right]_{q}$ code $\mathcal{C}_{4}$ exists by Theorem 2.5 , and an optimal $\left[\mathcal{F}_{3}, 3,1\right]_{q}$ code $\mathcal{C}_{3}$ is trivial. 
The above procedure from $\mathcal{F}$ to $\mathcal{F}^{*}$ yields a natural bijection $\psi$ from $\mathcal{F}$ to $\mathcal{F}^{*}$ (note that $\psi(3,3)=(2,0))$. For each $\boldsymbol{D} \in \mathcal{C}^{*}$, construct $a 10 \times 8$ matrix $\boldsymbol{C}_{\boldsymbol{D}}$ such that

$$
\boldsymbol{C}_{\boldsymbol{D}}(i, j)=\left\{\begin{array}{l}
\boldsymbol{D}(\psi(i, j)), \text { if }(i, j) \in \mathcal{F} \\
0, \text { otherwise }
\end{array}\right.
$$

Let $\mathcal{C}=\left\{\boldsymbol{C}_{\boldsymbol{D}}: \boldsymbol{D} \in \mathcal{C}^{*}\right\}$. Then $\mathcal{C}$ is an optimal $[\mathcal{F}, 10,4]_{q}$ code. Clearly $\mathcal{C}$ and $\mathcal{C}^{*}$ have the same linearity and dimension. The optimality is guaranteed by Lemma 2.2. It suffices to prove that for each nonzero $\boldsymbol{C}_{\boldsymbol{D}} \in \mathcal{C}, \operatorname{rank}\left(\boldsymbol{C}_{\boldsymbol{D}}\right) \geq 4$.

Clearly, $\operatorname{rank}\left(\boldsymbol{C}_{\boldsymbol{D}}\right) \geq \operatorname{rank}\left(\left.\boldsymbol{C}_{\boldsymbol{D}}\right|_{\mathcal{F}_{1}}\right)+\operatorname{rank}\left(\left.\boldsymbol{C}_{\boldsymbol{D}}\right|_{\mathcal{F}_{2}}\right)+\operatorname{rank}\left(\left.\boldsymbol{C}_{\boldsymbol{D}}\right|_{\mathcal{F}_{3}}\right) . \quad$ By Lemma 4.4, $\operatorname{rank}\left(\left.\boldsymbol{C}_{\boldsymbol{D}}\right|_{\mathcal{F}_{1}}\right)+\operatorname{rank}\left(\left.\boldsymbol{C}_{\boldsymbol{D}}\right|_{\mathcal{F}_{2}}\right) \geq \operatorname{rank}\left(\left.\boldsymbol{D}\right|_{\mathcal{F}_{12}}\right)$. Since $\operatorname{rank}\left(\left.\boldsymbol{C}_{\boldsymbol{D}}\right|_{\mathcal{F}_{3}}\right)=\operatorname{rank}\left(\left.\boldsymbol{D}\right|_{\mathcal{F}_{3}}\right)$, we have that $\operatorname{rank}\left(\boldsymbol{C}_{\boldsymbol{D}}\right) \geq \operatorname{rank}\left(\left.\boldsymbol{D}\right|_{\mathcal{F}_{12}}\right)+\operatorname{rank}\left(\left.\boldsymbol{D}\right|_{\mathcal{F}_{3}}\right)$. Note that $\left.\boldsymbol{D}\right|_{\mathcal{F}_{12}}=\boldsymbol{O}$ if and only if $\left.\boldsymbol{D}\right|_{\mathcal{F}_{3}}=\boldsymbol{O}$. If $\left.\boldsymbol{D}\right|_{\mathcal{F}_{12}} \neq \boldsymbol{O}$, then since $\mathcal{C}_{12}$ is an $\left[\mathcal{F}_{12}, 3,3\right]_{q}$ code, $\operatorname{rank}\left(\left.\boldsymbol{D}\right|_{\mathcal{F}_{12}}\right) \geq 3$, and since $\mathcal{C}_{3}$ is an $\left[\mathcal{F}_{3}, 3,1\right]_{q}$ code, $\operatorname{rank}\left(\left.\boldsymbol{D}\right|_{\mathcal{F}_{3}}\right) \geq 1$. So rank $\left(\boldsymbol{C}_{\boldsymbol{D}}\right) \geq 4$. If $\left.\boldsymbol{D}\right|_{\mathcal{F}_{12}}=\boldsymbol{O}$, then $\operatorname{rank}\left(\boldsymbol{C}_{\boldsymbol{D}}\right)=\operatorname{rank}\left(\left.\boldsymbol{C}_{\boldsymbol{D}}\right|_{\mathcal{F}_{4}}\right)=\operatorname{rank}\left(\left.\boldsymbol{D}\right|_{\mathcal{F}_{4}}\right)$. Since $\mathcal{C}_{4}$ is an $\left[\mathcal{F}_{4}, 7,4\right]_{q} \operatorname{code}$, $\operatorname{rank}\left(\left.\boldsymbol{D}\right|_{\mathcal{F}_{4}}\right) \geq 4$. Therefore, $\operatorname{rank}\left(\boldsymbol{C}_{\boldsymbol{D}}\right) \geq 4$.

Remark 4.6 No known construction can be applied to obtain Example 4.5. By Remark 3.2, all theorems in Section 2.3 .1 are invalid. Theorem 2.9 can provide an $[\mathcal{F}, 9,4]_{q}$ code for any prime power $q \geq 4$, but it is not optimal. Theorem 2.12 is also invalid. Otherwise, to get the required dimension $10, \mathcal{F}_{2}$ must contain the $7 \times 5$ Ferrers diagram in the lower right corner of $\mathcal{F}$ as its sub-Ferrers diagram. But then one cannot find $\mathcal{F}_{1}$ with at least 10 dots.

Let us now generalize Example 4.5.

\section{Construction 4.7 Let}

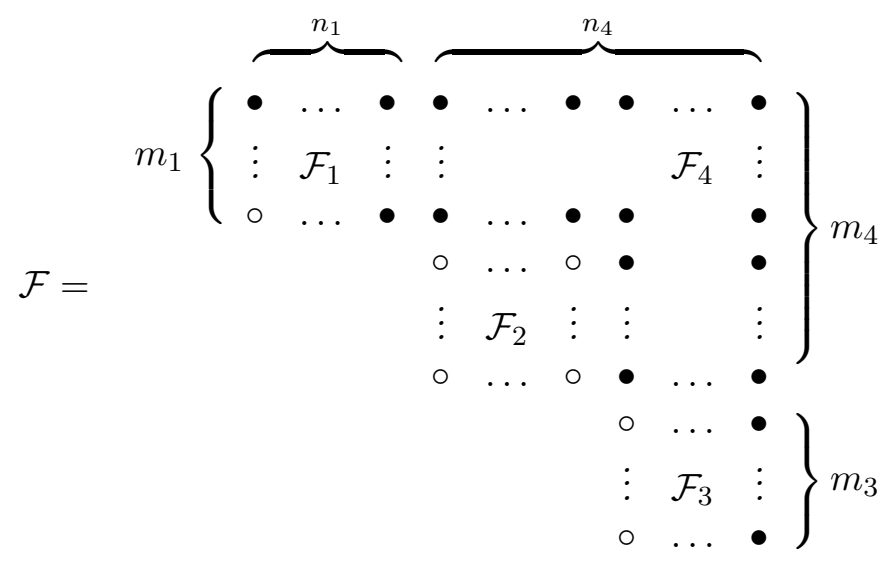

be an $m \times n$ Ferrers diagram, where $\mathcal{F}_{i}$ is an $m_{i} \times n_{i}$ Ferrers sub-diagram, $1 \leq i \leq 4$, satisfying that $m=m_{3}+m_{4}, n=n_{1}+n_{4}, m_{4} \geq m_{1}+m_{2}$ and $n_{4} \geq n_{2}+n_{3}$. Suppose that $\mathcal{F}_{12}$ is a proper combination of $\mathcal{F}_{1}$ and $\mathcal{F}_{2}$, and $\mathcal{C}_{12}$ is an $\left[\mathcal{F}_{12}, k_{1}, \delta_{1}\right]_{q}$ code. If there exist an $\left[\mathcal{F}_{3}, k_{3}, \delta_{3}\right]_{q}$ code $\mathcal{C}_{3}$ and an $\left[\mathcal{F}_{4}, k_{4}, \delta_{4}\right]_{q}$ code $\mathcal{C}_{4}$, then there exists an $[\mathcal{F}, k, \delta]_{q}$ code $\mathcal{C}$, where $k=\min \left\{k_{1}, k_{3}\right\}+k_{4}, \delta=\min \left\{\delta_{1}+\delta_{3}, \delta_{4}\right\}$. Note that the dots "•" in $\mathcal{F}$ have to exist, whereas the dots " $\circ$ " can exist or not.

Proof Construct a new Ferrers diagram 


$$
\mathcal{F}^{*}=\left(\begin{array}{cc}
\mathcal{F}_{12} & \hat{\mathcal{F}}_{4} \\
& \mathcal{F}_{3}
\end{array}\right)
$$

where $\hat{\mathcal{F}}_{4}$ is obtained by adding the fewest number of new dots to the lower-left corner of $\mathcal{F}_{4}$ such that $\mathcal{F}^{*}$ is a Ferrers diagram. Obviously, an $\left[\hat{\mathcal{F}}_{4}, k_{4}, \delta_{4}\right]_{q}$ code can be obtained by adding all-zero rows to matrices in $\mathcal{C}_{4}$. It follows that by Theorem 4.1, an $\left[\mathcal{F}^{*}, k, \delta\right]_{q}$ code $\mathcal{C}^{*}$ exists, where $k=\min \left\{k_{1}, k_{3}\right\}+k_{4}, \delta=\min \left\{\delta_{1}+\delta_{3}, \delta_{4}\right\}$.

The above procedure from $\mathcal{F}$ to $\mathcal{F}^{*}$ yields a natural injection $\psi$ from $\mathcal{F}$ to $\mathcal{F}^{*}$. Now, for each $\boldsymbol{D} \in \mathcal{C}^{*}$, construct an $m \times n$ matrix $\boldsymbol{C}_{\boldsymbol{D}}$ such that

$$
\boldsymbol{C}_{\boldsymbol{D}}(i, j)=\left\{\begin{array}{l}
\boldsymbol{D}(\psi(i, j)), \text { if }(i, j) \in \mathcal{F} \\
0, \quad \text { otherwise }
\end{array}\right.
$$

Let $\mathcal{C}=\left\{\boldsymbol{C}_{\boldsymbol{D}}: \boldsymbol{D} \in \mathcal{C}^{*}\right\}$. It is readily checked that $\mathcal{C}$ is an $[\mathcal{F}, k, \delta]_{q}$ code.

When $\mathcal{F}_{2}$ is empty, Construction 4.7 yields Theorem 4.1 .

\subsection{Relaxation of dimensions}

Construction 4.7 produces an $\left[\mathcal{F}, \min \left\{k_{1}, k_{3}\right\}+k_{4}, \delta\right]_{q}$ code from an $\left[\mathcal{F}_{12}, k_{1}, \delta_{1}\right]_{q}$ code $\mathcal{C}_{12}$, an $\left[\mathcal{F}_{3}, k_{3}, \delta_{3}\right]_{q}$ code $\mathcal{C}_{3}$ and an $\left[\mathcal{F}_{4}, k_{4}, \delta_{4}\right]_{q}$ code $\mathcal{C}_{4}$. This procedure doesn't make full use of dimensions of $\mathcal{C}_{12}$ and $\mathcal{C}_{3}$. We hope to find a proper combination $\mathcal{F}^{* *}$ of $\mathcal{F}_{12}$ and $\mathcal{F}_{3}$ such that there exists an $\left[\mathcal{F}^{* *}, k^{\prime}, \delta^{\prime}\right]_{q}$ code, where $k^{\prime}>\min \left\{k_{1}, k_{3}\right\}$. The following construction provides a possible way to realize our idea. We start from an example.

Example 4.8 We here construct an optimal $[\mathcal{F}, 13,4]_{q}$ code $\mathcal{C}$ for any prime power $q$, where

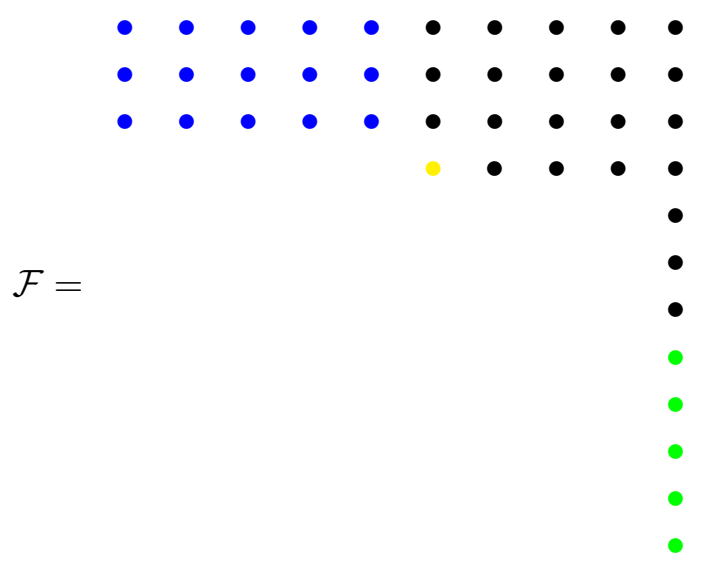

First take the following four Ferrers sub-diagrams of $\mathcal{F}$ :

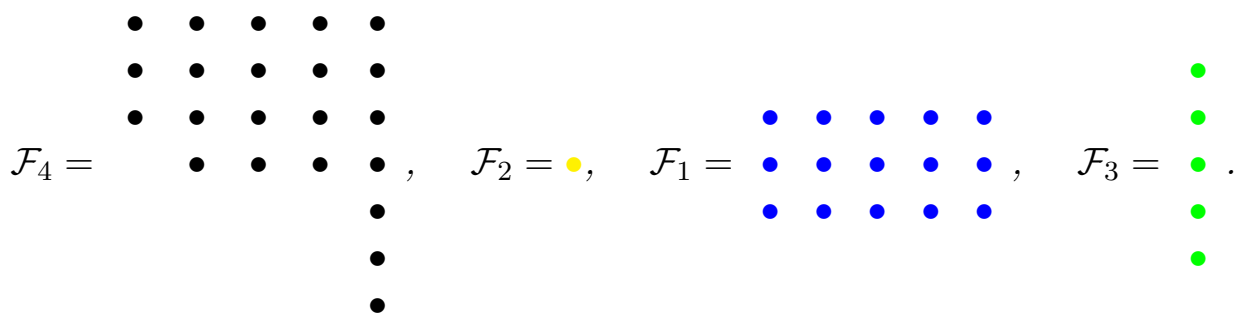


Then take a proper combination $\mathcal{F}_{123}$ of $\mathcal{F}_{1}, \mathcal{F}_{2}$ and $\mathcal{F}_{3}$ on mappings $\phi_{1}, \phi_{2}$ and $\phi_{3}$ as follows

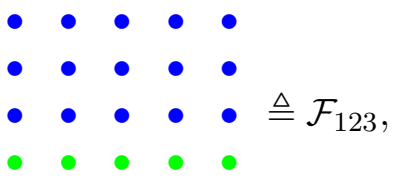

where $\phi_{1}: \mathcal{F}_{1} \longrightarrow \mathcal{F}_{123}$ satisfies $\phi_{1}(i, j)=(i, j)$ for any $(i, j) \in[3] \times[5], \phi_{2}: \mathcal{F}_{2} \longrightarrow \mathcal{F}_{123}$ satisfies $\phi_{2}(0,0)=(4,4)$, and $\phi_{3}: \mathcal{F}_{3} \longrightarrow \mathcal{F}_{123}$ satisfies $\phi_{3}(j, 0)=(3, j)$ for any $j \in[5]$. By Theorem 2.3, there exists an optimal $\left[\mathcal{F}_{123}^{t}, 6,4\right]_{q}$ code, which implies an optimal $\left[\mathcal{F}_{123}, 6,4\right]_{q}$ code $\mathcal{C}_{123}$. By Theorem $\left[2.5\right.$, there exists an optimal $\left[\mathcal{F}_{4}, 7,4\right]_{q}$ code $\mathcal{C}_{4}$.

The above procedure yields two natural bijection $\psi_{1}:\left.\mathcal{F}\right|_{\mathcal{F}_{1}, \mathcal{F}_{2}, \mathcal{F}_{3}} \longrightarrow \mathcal{F}_{123}$ and $\psi_{2}$ : $\left.\mathcal{F}\right|_{\mathcal{F}_{4}} \longrightarrow \mathcal{F}_{4}$. For each $\boldsymbol{B} \in \mathcal{C}_{123}$ and $\boldsymbol{D} \in \mathcal{C}_{4}$, construct a $12 \times 10$ matrix $\boldsymbol{C}_{\boldsymbol{B}, \boldsymbol{D}}$ such that

$$
\boldsymbol{C}_{\boldsymbol{B}, \boldsymbol{D}}(i, j)= \begin{cases}\boldsymbol{B}\left(\psi_{1}(i, j)\right), & \text { if }\left.(i, j) \in \mathcal{F}\right|_{\mathcal{F}_{1}, \mathcal{F}_{2}, \mathcal{F}_{3}} \\ \boldsymbol{D}\left(\psi_{2}(i, j)\right), & \text { if }\left.(i, j) \in \mathcal{F}\right|_{\mathcal{F}_{4}} ; \\ 0, & \text { if }(i, j) \notin \mathcal{F} .\end{cases}
$$

Let $\mathcal{C}=\left\{\boldsymbol{C}_{\boldsymbol{B}, \boldsymbol{D}}: \boldsymbol{B} \in \mathcal{C}_{123}, \boldsymbol{D} \in \mathcal{C}_{4}\right\}$. Then $\mathcal{C}$ is an optimal $[\mathcal{F}, 13,4]_{q}$ code. Clearly $\mathcal{C}$ is a code in $\mathcal{F}$ of dimension 13. The optimality is guaranteed by Lemma 2.2 . It suffices to prove that for each nonzero $\boldsymbol{C}_{\boldsymbol{B}, \boldsymbol{D}} \in \mathcal{C}, \operatorname{rank}\left(\boldsymbol{C}_{\boldsymbol{B}, \boldsymbol{D}}\right) \geq 4$.

Clearly, $\operatorname{rank}\left(\boldsymbol{C}_{\boldsymbol{B}, \boldsymbol{D}}\right) \geq \operatorname{rank}\left(\left.\boldsymbol{C}_{\boldsymbol{B}, \boldsymbol{D}}\right|_{\mathcal{F}_{1}}\right)+\operatorname{rank}\left(\left.\boldsymbol{C}_{\boldsymbol{B}, \boldsymbol{D}}\right|_{\mathcal{F}_{2}}\right)+\operatorname{rank}\left(\left.\boldsymbol{C}_{\boldsymbol{B}, \boldsymbol{D}}\right|_{\mathcal{F}_{3}}\right) \geq \operatorname{rank}(\boldsymbol{B})$, where the second inequality comes from Lemma 4.4. If $\boldsymbol{B} \neq \boldsymbol{O}$, then since $\mathcal{C}_{123}$ is an $\left[\mathcal{F}_{123}, 6,4\right]_{q}$ code, $\operatorname{rank}(\boldsymbol{B}) \geq 4$. If $\boldsymbol{B}=\boldsymbol{O}$, then $\operatorname{rank}\left(\boldsymbol{C}_{\boldsymbol{B}, \boldsymbol{D}}\right)=\operatorname{rank}\left(\boldsymbol{C}_{\boldsymbol{B}, \boldsymbol{D}} \mid \mathcal{F}_{4}\right)=$ $\operatorname{rank}(\boldsymbol{D})$. Since $\mathcal{C}_{4}$ is an $\left[\mathcal{F}_{4}, 7,4\right]_{q}$ code and $\boldsymbol{D} \neq 0$, we have rank $(\boldsymbol{D}) \geq 4$. Therefore, $\operatorname{rank}\left(\boldsymbol{C}_{\boldsymbol{B}, \boldsymbol{D}}\right) \geq 4$.

Remark 4.9 No known construction can be applied to obtain Example 4.8. By Remark 3.2. all theorems in Section 2.3.1 are invalid. Theorem 2.9 can provide an $[\mathcal{F}, 11,4]_{q}$ code for any prime power $q \geq 4$, but it is not optimal. Theorem 2.12 is also invalid. Otherwise, to get the required dimension $13, \mathcal{F}_{2}$ must be the $9 \times 5$ Ferrers diagram in the lower right corner of $\mathcal{F}$, and $\mathcal{F}_{1}$ must be the $3 \times 5$ Ferrers diagram in the top left corner of $\mathcal{F}$. Then $\delta_{1}=\delta_{2}=1$ because of the dimension 13 , which contradicts with $\delta=4$.

Let us now generalize Example 4.8 .

Construction 4.10 Let

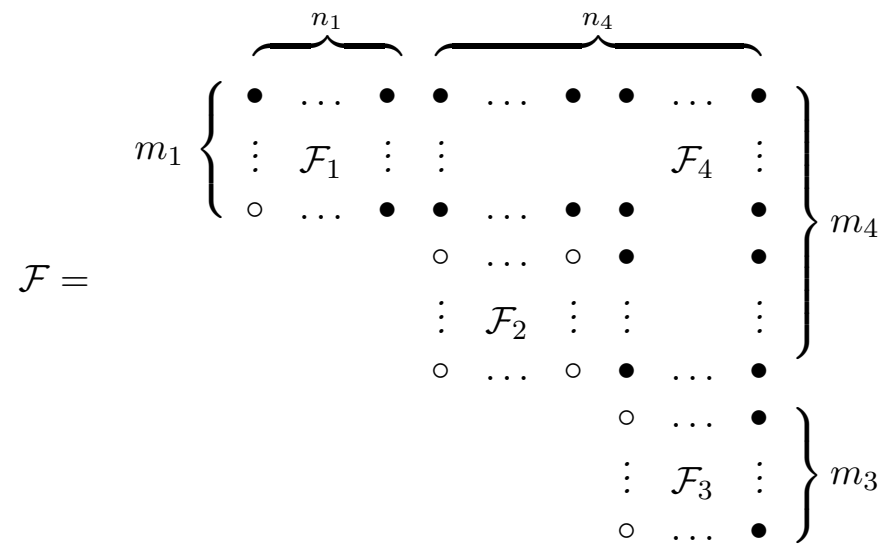


be an $m \times n$ Ferrers diagram, where $\mathcal{F}_{i}$ is an $m_{i} \times n_{i}$ Ferrers sub-diagram, $1 \leq i \leq 4$, satisfying that $m=m_{3}+m_{4}, n=n_{1}+n_{4}, m_{4} \geq m_{1}+m_{2}$ and $n_{4} \geq n_{2}+n_{3}$. Suppose that $\mathcal{F}_{123}$ is a proper combination of $\mathcal{F}_{1}, \mathcal{F}_{2}$ and $\mathcal{F}_{3}$, and $\mathcal{C}_{123}$ is an $\left[\mathcal{F}_{123}, k_{1}, \delta_{1}\right]_{q}$ code. If there exists an $\left[\mathcal{F}_{4}, k_{4}, \delta_{4}\right]_{q}$ code $\mathcal{C}_{4}$, then there exists an $\left[\mathcal{F}, k_{1}+k_{4}, \delta\right]_{q}$ code $\mathcal{C}$, where $\delta=\min \left\{\delta_{1}, \delta_{4}\right\}$.

Proof Take two natural bijections $\psi_{1}:\left.\mathcal{F}\right|_{\mathcal{F}_{1}, \mathcal{F}_{2}, \mathcal{F}_{3}} \longrightarrow \mathcal{F}_{123}$ and $\psi_{2}:\left.\mathcal{F}\right|_{\mathcal{F}_{4}} \longrightarrow \mathcal{F}_{4}$. For each $\boldsymbol{B} \in \mathcal{C}_{123}$ and $\boldsymbol{D} \in \mathcal{C}_{4}$, construct an $m \times n$ matrix $\boldsymbol{C}_{\boldsymbol{B}, \boldsymbol{D}}$ such that

$$
\boldsymbol{C}_{\boldsymbol{B}, \boldsymbol{D}}(i, j)= \begin{cases}\boldsymbol{B}\left(\psi_{1}(i, j)\right), & \text { if }\left.(i, j) \in \mathcal{F}\right|_{\mathcal{F}_{1}, \mathcal{F}_{2}, \mathcal{F}_{3}} \\ \boldsymbol{D}\left(\psi_{2}(i, j)\right), & \text { if }\left.(i, j) \in \mathcal{F}\right|_{\mathcal{F}_{4}} \\ 0, & \text { if }(i, j) \notin \mathcal{F}\end{cases}
$$

Let $\mathcal{C}=\left\{\boldsymbol{C}_{\boldsymbol{B}, \boldsymbol{D}}: \boldsymbol{B} \in \mathcal{C}_{123}, \boldsymbol{D} \in \mathcal{C}_{4}\right\}$. It is readily checked that $\mathcal{C}$ is an $\left[\mathcal{F}, k_{1}+k_{4}, \delta\right]_{q}$ code $\mathcal{C}$, where $\delta=\min \left\{\delta_{1}, \delta_{4}\right\}$.

Remark 4.11 Compared with Construction 4.7, Construction 4.10 starts from a proper combination $\mathcal{F}_{123}$ of $\mathcal{F}_{1}, \mathcal{F}_{2}$ and $\mathcal{F}_{3}$, which must be a Ferrers diagram according to the definition of proper combinations. This requirement sometimes restricts the value of rank of the resulting code $\mathcal{C}$. For example, in Example 4.5, the proper combination of $\mathcal{F}_{12}$ and $\mathcal{F}_{3}$ will provide codes with rank at most 3 , while the required code has rank 4.

Theorem 4.12 Let $\delta \leq y \leq \min \{m-\delta+2, n-\delta-1\}$. Let

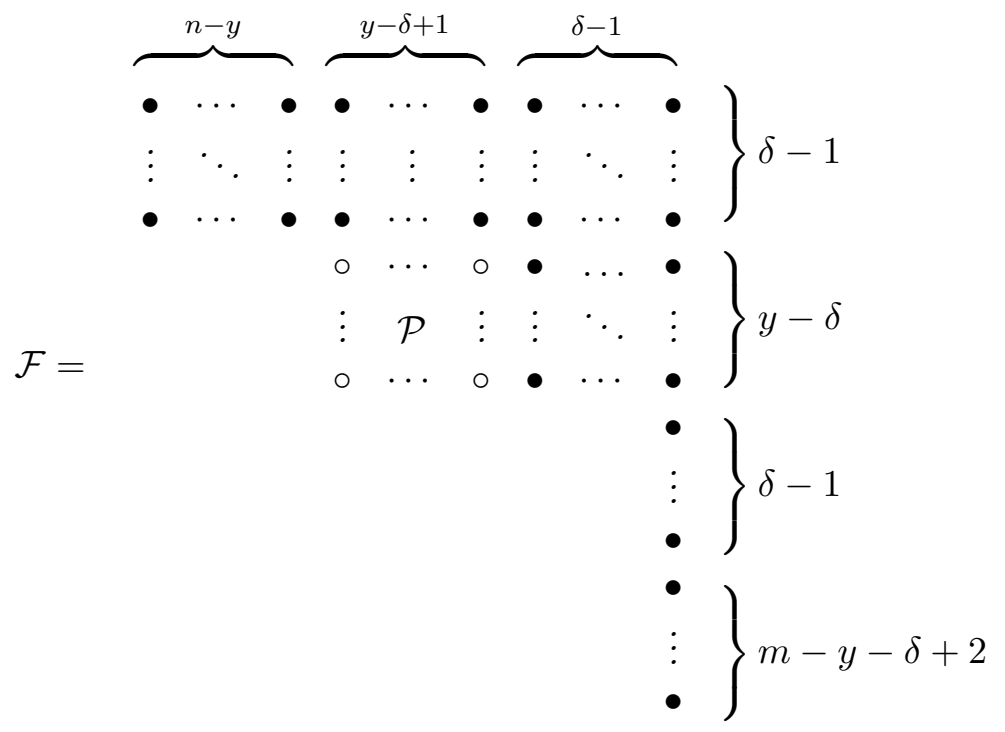

be an $m \times n$ Ferrers diagram $\mathcal{F}$. Let $z_{i}$ be the number of dots in the $i$-th column of $\mathcal{P}$, $i \in[y-\delta+1]$. If $z_{0} \leq n-y$, then Construction 4.10 provides an optimal $[\mathcal{F}, k, \delta]_{q}$ code, where

$$
k=\left\{\begin{array}{l}
m-y+1+(y-\delta)(\delta-1)+|\mathcal{P}|, \quad \text { if } m-n \leq \delta-2 \\
n-1+(y-\delta)(\delta-2)+|\mathcal{P}|, \quad \text { otherwise }
\end{array}\right.
$$

Proof Let $\mathcal{P}_{1}$ denote the Ferrers diagram obtained by removing the first column of $\mathcal{P}$. Consider the following four Ferrers sub-diagrams of $\mathcal{F}$ : 


$$
\mathcal{F}_{1}=\overbrace{\left.\begin{array}{ccc}
\bullet & \cdots & \bullet \\
\vdots & \ddots & \vdots \\
\bullet & \cdots & \bullet
\end{array}\right\} \delta-1}^{n-y}, \quad \mathcal{F}_{2}=\begin{array}{l}
0 \\
\vdots \\
\circ
\end{array}\} z_{0}, \quad \mathcal{F}_{3}=\begin{array}{l}
\bullet \\
\vdots
\end{array}\} m-y-\delta+2,
$$

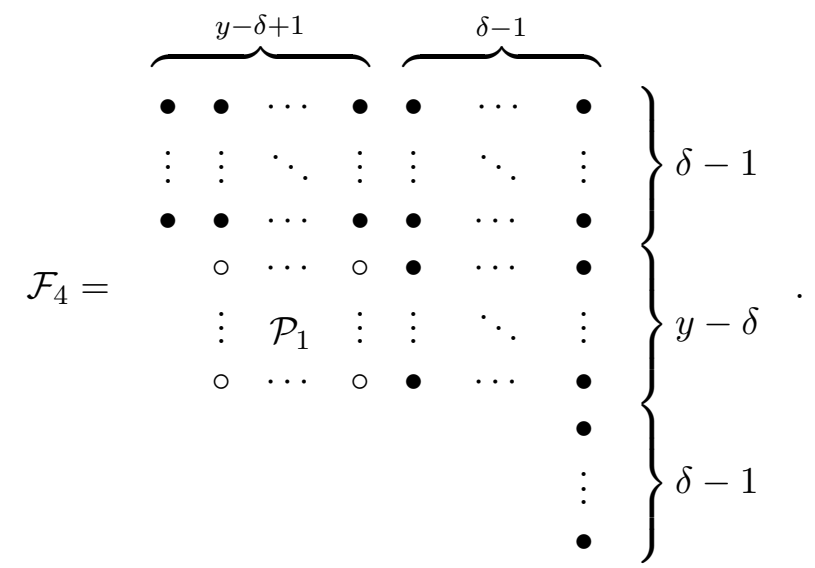

By Theorem [2.5, there exists an $\left[\mathcal{F}_{4},(y-\delta+1)(\delta-1)-z_{0}+|\mathcal{P}|, \delta\right]_{q}$ code.

If $n \geq m-\delta+2$, then $n-y \geq m-\delta+2-y$. When $m-y-\delta+2 \geq z_{0}$, take a proper combination $\mathcal{F}_{123}$ of $\mathcal{F}_{1}, \mathcal{F}_{2}$ and $\mathcal{F}_{3}$ as follows (note that $z_{0} \leq n-y$ by assumption)

$$
\mathcal{F}_{123}=\left(\begin{array}{c}
\mathcal{F}_{1} \\
\mathcal{F}_{3}^{t} \\
\mathcal{F}_{2}^{t}
\end{array}\right) \text {. }
$$

When $m-y-\delta+2<z_{0}$, take

$$
\mathcal{F}_{123}=\left(\begin{array}{c}
\mathcal{F}_{1} \\
\mathcal{F}_{2}^{t} \\
\mathcal{F}_{3}^{t}
\end{array}\right) .
$$

$\mathcal{F}_{123}$ is an $(\delta+1) \times(n-y)$ Ferrers diagram. By assumption, $n-y \geq \delta+1$, so by Theorem 2.3. there exists an $\left[\mathcal{F}_{123}, m-y-\delta+2+z_{0}, \delta\right]_{q}$ code. Then apply Construction 4.10 to obtain an $[\mathcal{F}, m-y+1+(y-\delta)(\delta-1)+|\mathcal{P}|, \delta]_{q}$ code, which is optimal by Lemma 2.2 (one can check it by counting the number of dots in $\mathcal{F}$ which are not contained in the first $\delta-1$ rows).

If $n<m-\delta+2$, then $n-y<m-\delta+2-y$. Take a proper combination $\mathcal{F}_{123}$ of $\mathcal{F}_{1}$, $\mathcal{F}_{2}$ and $\mathcal{F}_{3}$ as follows

$$
\mathcal{F}_{123}=\left(\begin{array}{c}
\mathcal{F}_{3}^{t} \\
\mathcal{F}_{1} \\
\mathcal{F}_{2}^{t}
\end{array}\right) \text {. }
$$

$\mathcal{F}_{123}$ is an $(\delta+1) \times(m-y-\delta+2)$ Ferrers diagram. By assumption, $n-y \geq \delta+1$, so $m-y-\delta+2>\delta+1$. Thus by Theorem 2.3 , there exists an $\left[\mathcal{F}_{123}, n-y+z_{0}, \delta\right]_{q}$ code. Then apply Construction 4.10 to obtain an $[\mathcal{F}, n-1+(y-\delta)(\delta-2)+|\mathcal{P}|, \delta]_{q}$ code, which is optimal by Lemma 2.2 (one can check it by counting the number of dots in $\mathcal{F}$ which are not contained in the first $\delta-2$ rows and the rightmost column).

We remark that Theorem 4.12 with $n=10, y=5, m=12, \delta=4, k=13$ and $\mathcal{P}=\bullet$ • yields Example 4.8 . 


\subsection{A special case: $\mathcal{F}_{2}$ having only one dot}

Constructions 4.7 and 4.10 require that $\mathcal{F}_{2}$ doesn't contain any dots of $\mathcal{F}$ in the first $n_{1}$ columns and the last $m_{3}$ rows. However, when $\mathcal{F}_{2}$ contains only one dot, this restriction can be relaxed.

Construction 4.13 Let $m=m_{1}+m_{3}$ and $n=n_{1}+n_{3}$. Let

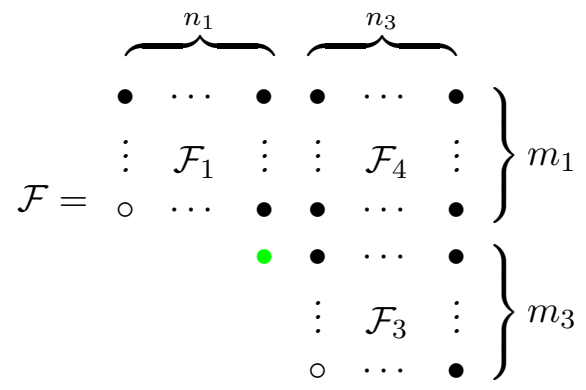

be an $m \times n$ Ferrers diagram, where $\mathcal{F}_{1}$ is an $m_{1} \times n_{1}$ Ferrers diagram, $\mathcal{F}_{2}=\bullet, \mathcal{F}_{3}$ is an $m_{3} \times n_{3}$ Ferrers diagram, and $\mathcal{F}_{4}$ is an $m_{1} \times n_{3}$ full Ferrers diagram. Sort the list $\{1\} \cup\left\{\rho_{i}\left(\mathcal{F}_{1}\right): i \in\left[m_{1}\right]\right\} \cup\left\{\gamma_{j}\left(\mathcal{F}_{3}\right): j \in\left[n_{3}\right]\right\}$ from small to large, where $\rho_{i}\left(\mathcal{F}_{1}\right)$ denotes the number of dots in the $i$-th row of $\mathcal{F}_{1}$ and $\gamma_{j}\left(\mathcal{F}_{3}\right)$ denotes the number of dots in the $j$-th column of $\mathcal{F}_{3}$. The elements in the sorted list are rewritten as $\alpha_{0} \leq \alpha_{1} \leq \ldots \leq \alpha_{m_{1}+n_{3}}$. Suppose that $\mathcal{F}_{123}$ is a proper combination of $\mathcal{F}_{1}, \mathcal{F}_{2}$ and $\mathcal{F}_{3}$ satisfying $\gamma_{l}\left(\mathcal{F}_{123}\right)=\alpha_{l}$ for $l \in\left[m_{1}+n_{3}+1\right]$, where $\gamma_{l}\left(\mathcal{F}_{123}\right)$ denotes the number of dots in the l-th column of $\mathcal{F}_{123}$, and $\mathcal{C}_{123}$ is an $\left[\mathcal{F}_{123}, k_{1}, \delta_{1}\right]_{q}$ code. If there exists an $\left[\mathcal{F}_{4}, k_{4}, \delta_{4}\right]_{q}$ code $\mathcal{C}_{4}$, then there exists an $\left[\mathcal{F}, k_{1}+k_{4}, \delta\right]_{q}$ code $\mathcal{C}$, where $\delta=\min \left\{\delta_{1}, \delta_{4}\right\}$.

Proof Take a natural bijection $\psi_{1}:\left.\mathcal{F}\right|_{\mathcal{F}_{1}, \mathcal{F}_{2}, \mathcal{F}_{3}} \longrightarrow \mathcal{F}_{123}$ such that $\psi_{1}\left(m_{1}, n_{1}-1\right)=$ $(0,0), \psi_{1}\left(i, n_{1}-1\right)=(0, *)$ for each $i \in\left[m_{1}\right]$, and $\psi_{1}\left(m_{1}, j\right)=(0, *)$ for each $n_{1} \leq j \leq$ $n_{1}+n_{3}-1$. Take a natural bijection $\psi_{2}:\left.\mathcal{F}\right|_{\mathcal{F}_{4}} \longrightarrow \mathcal{F}_{4}$. For each $\boldsymbol{B} \in \mathcal{C}_{123}$ and $\boldsymbol{D} \in \mathcal{C}_{4}$, construct an $m \times n$ matrix $\boldsymbol{C}_{\boldsymbol{B}, \boldsymbol{D}}$ such that

$$
\boldsymbol{C}_{\boldsymbol{B}, \boldsymbol{D}}(i, j)= \begin{cases}\boldsymbol{B}\left(\psi_{1}(i, j)\right), & \text { if }\left.(i, j) \in \mathcal{F}\right|_{\mathcal{F}_{1}, \mathcal{F}_{2}, \mathcal{F}_{3}} \\ \boldsymbol{D}\left(\psi_{2}(i, j)\right), & \text { if }\left.(i, j) \in \mathcal{F}\right|_{\mathcal{F}_{4}} \\ 0, & \text { if }(i, j) \notin \mathcal{F} .\end{cases}
$$

Let $\mathcal{C}=\left\{\boldsymbol{C}_{\boldsymbol{B}, \boldsymbol{D}}: \boldsymbol{B} \in \mathcal{C}_{123}, \boldsymbol{D} \in \mathcal{C}_{4}\right\}$. Then $\mathcal{C}$ is an $\left[\mathcal{F}, k_{1}+k_{4}, \delta\right]_{q}$ code $\mathcal{C}$, where $\delta=\min \left\{\delta_{1}, \delta_{4}\right\}$.

One can easily verify the linearity and the dimension of the code. It suffices to examine the minimum rank weight of any nonzero codewords $\boldsymbol{C}_{\boldsymbol{B}, \boldsymbol{D}}$ from $\mathcal{C}$. We give a sketch of the counting for ranks below. The technique is similar to that in Example 4.8.

Let

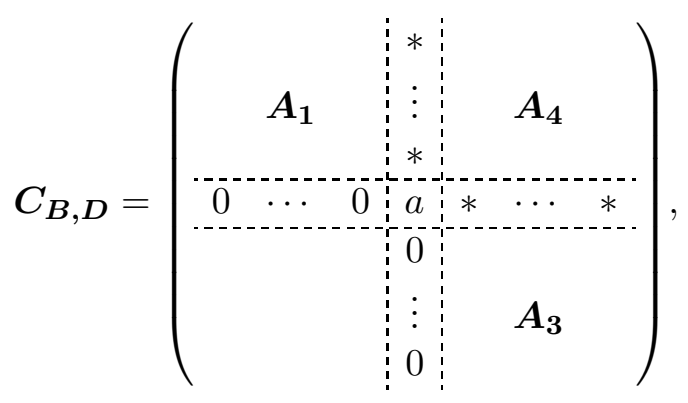


where $a$ corresponds to the $\operatorname{dot}$ in $\mathcal{F}_{2}$. If $\boldsymbol{A}_{\mathbf{4}} \neq \boldsymbol{O}$, then since $\mathcal{C}_{4}$ is an $\left[\mathcal{F}_{4}, k_{4}, \delta_{4}\right]_{q}$ code, $\operatorname{rank}\left(\boldsymbol{C}_{\boldsymbol{B}, \boldsymbol{D}}\right) \geq \operatorname{rank}\left(\boldsymbol{A}_{\mathbf{4}}\right)=\operatorname{rank}(\boldsymbol{D}) \geq \delta_{4}$. If $\boldsymbol{A}_{\mathbf{4}}=\boldsymbol{O}$ and $a=0$, then since $\mathcal{C}_{123}$ is an $\left[\mathcal{F}_{123}, k_{1}, \delta_{1}\right]_{q} \operatorname{code}, \operatorname{rank}\left(\boldsymbol{C}_{\boldsymbol{B}, \boldsymbol{D}}\right)=\operatorname{rank}\left(\left.\boldsymbol{C}_{\boldsymbol{B}, \boldsymbol{D}}\right|_{\mathcal{F}_{1}}\right)+\operatorname{rank}\left(\boldsymbol{C}_{\boldsymbol{B}, \boldsymbol{D}} \mid \mathcal{F}_{3}\right) \geq \operatorname{rank}(\mathbf{B}) \geq \delta_{1}$. If $\boldsymbol{A}_{\mathbf{4}}=\boldsymbol{O}$ and $a \neq 0$, then $\operatorname{rank}\left(\boldsymbol{C}_{\boldsymbol{B}, \boldsymbol{D}}\right) \geq \operatorname{rank}\left(\boldsymbol{A}_{\mathbf{1}}\right)+1+\operatorname{rank}\left(\boldsymbol{A}_{\mathbf{3}}\right)$. According to $\psi_{1}, \mathbf{B}$ is of the form (a permutation of columns are allowed)

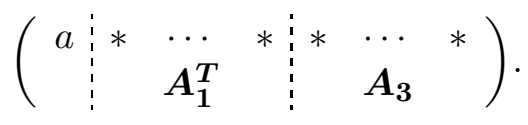

Since $\operatorname{rank}\left(\boldsymbol{A}_{\mathbf{1}}\right)+1+\operatorname{rank}\left(\boldsymbol{A}_{\mathbf{3}}\right) \geq \operatorname{rank}(\boldsymbol{B})$, we have $\operatorname{rank}\left(\boldsymbol{C}_{\boldsymbol{B}, \boldsymbol{D}}\right) \geq \delta_{1}$.

Theorem 4.14 Take $\delta_{1}=\delta_{4}=\delta$ in Construction 4.13 such that $\delta \leq m_{1}+1$. Suppose that $\mathcal{F}$ in Construction 4.13 satisfies:

(1) if $\delta<m_{1}+1$, then $n_{3} \geq m_{1}$;

(2) $1+m_{1}+n_{3} \leq \max \left\{n_{1}, m_{3}\right\}$;

(3) $\alpha_{m_{1}+n_{3}-\delta+2} \geq m_{1}+n_{3}$;

(4) $\rho_{\delta-2}-n_{3} \geq m_{3}$,

where $\rho_{i}$ denotes the number of dots in the $i$-th row of $\mathcal{F}, i \in\left[m_{1}+m_{3}\right]$. Then there exists an optimal $\left[\mathcal{F}, \sum_{i=\delta-1}^{m_{1}+m_{3}-1} \rho_{i}, \delta\right]_{q}$ code $\mathcal{C}$ for any prime power $q$.

Proof By Theorem 2.3 , due to Condition (1), there is an optimal $\left[\mathcal{F}_{4}, n_{3}\left(m_{1}-\delta+1\right), \delta\right]_{q}$ code $\mathcal{C}_{4}$ for any prime power $q$. Note that when $\delta=m_{1}+1$, it consists of only a zero codeword.

Note that $\mathcal{F}_{123}$ has $m_{1}+n_{3}+1$ columns. By Theorem 2.5, due to Conditions (2) and $(3)$, there is an $\left[\mathcal{F}_{123}, \sum_{i=0}^{m_{1}+n_{3}-\delta+1} \alpha_{i}, \delta\right]_{q}$ code $\mathcal{C}_{123}$ for any prime power $q$, where $\alpha_{i}$ denotes the number of dots in the $i$-th column of $\mathcal{F}_{123}$. It is optimal by Lemma 2.2 . Condition (4) ensures all dots in $\mathcal{F}_{3}$ contribute dimensions for $\mathcal{C}_{123}$, so $\sum_{i=0}^{m_{1}+n_{3}-\delta+1} \alpha_{i}=$ $\sum_{i=\delta-1}^{m_{1}-1}\left(\rho_{i}-n_{3}\right)+\sum_{i=m_{1}}^{m_{1}+m_{3}-1} \rho_{i}$.

Therefore, we can apply Construction 4.13 to obtain an optimal $[\mathcal{F}, k, \delta]_{q}$ code, where $k=n_{3}\left(m_{1}-\delta+1\right)+\sum_{i=\delta-1}^{m_{1}-1}\left(\rho_{i}-n_{3}\right)+\sum_{i=m_{1}}^{m_{1}+m_{3}-1} \rho_{i}=\sum_{i=\delta-1}^{m_{1}+m_{3}-1} \rho_{i}$.

Example 4.15 Consider the following Ferrers diagram:

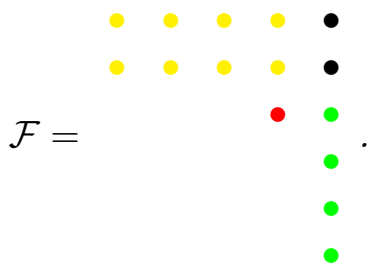

Let $\delta=3$ and

$$
\mathcal{F}_{1}=\bullet \quad, \mathcal{F}_{2}=\bullet, \mathcal{F}_{3}=\stackrel{\bullet}{\bullet}, \mathcal{F}_{4}=\bullet
$$


Then $m_{1}=2, n_{1}=4, m_{3}=4, n_{3}=1, \alpha_{0}=1$ and $\alpha_{i}=4$ for $i \in\{1,2,3\}$. So the conditions in Theorem 4.14 are satisfied, and we can construct an optimal $[\mathcal{F}, 5,3]$ code.

Remark 4.16 No known construction can be applied to obtain Example 4.15. By Remark 3.2 , all theorems in Section 2.3 .1 are invalid. Theorem 2.9 provides an $[\mathcal{F}, 4,3]_{q}$ code for any prime power $q \geq 3$, but it is not optimal. Theorem 2.12 is also invalid. Otherwise, to get the required dimension $4, \mathcal{F}_{2}$ must be the $4 \times 2$ Ferrers diagram in the lower right corner of $\mathcal{F}$, and $\mathcal{F}_{1}$ must be the $2 \times 3$ Ferrers diagram in the top left corner of $\mathcal{F}$. Then $\delta_{1}=2$ and $\delta_{2}=1$ because of the dimension 4 , which contradicts with $\delta=4$.

\section{Concluding remarks}

Main contributions of this paper lie in the following two aspects. One is to generalize Construction 2 in [5] by exploring subcodes of Gabidulin codes. Construction 2 in [5] requires that each of the rightmost $\delta-1$ columns in Ferrers diagram $\mathcal{F}$ has at least $n-1$ dots. We relax the condition $n-1$ to $n-r$ (see Theorem 3.13). The other is to generalize Theorem 9 in [5] by introducing the concept of proper combinations of Ferrers diagrams (see Constructions 4.7, 4.10 and 4.13). This is the first time constructions for FDRM codes with large size based on small ones are investigated systematically since they are introduced in [5].

Recently, a new family of MRD codes is presented in [16]. A natural question is how to use it to construct new optimal FDRM codes.

Another question is whether it is possible in some circumstances to require that

$\mathcal{F}_{1}$ and $\mathcal{F}_{2}$ in Construction 4.7 or $\mathcal{F}_{1}, \mathcal{F}_{2}$ and $\mathcal{F}_{3}$ in Construction 4.10 are not Ferrers diagrams.

\section{A Appendix}

Proof of Lemma 3.4 Let

$$
\mathbf{B}=\left(\begin{array}{llll}
1 & u_{0,1} & \cdots & u_{0, n-1} \\
& 1 & \cdots & u_{1, n-1} \\
& & \ddots & \vdots \\
& & & 1
\end{array}\right)
$$

Then

$\mathbf{G B}=\left(\begin{array}{ccccccc}1 & u_{0,1} & \ldots & u_{0, k-1} & u_{0, k}+a_{1, k} \beta^{k} & \ldots & u_{0, n-1}+\sum_{i=k}^{n-2} u_{i, n-1} a_{1, i} \beta^{i}+a_{1, n-1} \beta^{n} \\ & 1 & \cdots & u_{1, k-1} & u_{1, k}+a_{2, k} \beta^{k-1} & \cdots & u_{1, n-1}+\sum_{i=k}^{n-2} u_{i, n-1} a_{2, i} \beta^{i-1}+a_{2, n-1} \beta^{n-2} \\ & & \ddots & \vdots & \vdots & \ddots & \vdots \\ & & & 1 & u_{k-1, k}+a_{k, k} \beta & \cdots & u_{k-1, n-1}+\sum_{i=k}^{n-2} u_{i, n-1} a_{k, i} \beta^{i-k+1}+a_{k, n-1} \beta^{n-k}\end{array}\right)$.

Let $\mathbf{D}_{k}$ be any $k \times k$ submatrix of GB. Then $\operatorname{det}\left(\mathbf{D}_{k}\right)$ is a polynomial on $\beta$.

Case 1. $\mathbf{D}_{k}$ doesn't contain the last column of GB. If we could prove that the degree of $\operatorname{det}\left(\mathbf{D}_{k}\right)$ is less than $m$, and the leading coefficient of $\operatorname{det}\left(\mathbf{D}_{k}\right)$ is a minor of $\mathbf{A}_{1}$, then since every minor of $\mathbf{A}_{1}$ is nonzero, we would have $\operatorname{det}\left(\mathbf{D}_{k}\right) \neq 0$.

Subcase 1.1. $\mathbf{D}_{k}$ doesn't contain any of the first $k$ columns of GB. Take 


$$
\mathbf{M}_{1}=\left(\begin{array}{cccc}
a_{1, i_{1}} \beta^{i_{1}} & a_{1, i_{2}} \beta^{i_{2}} & \cdots & a_{1, i_{k}} \beta^{i_{k}} \\
a_{2, i_{1}} \beta^{i_{1}-1} & a_{1, i_{2}} \beta^{i_{2}-1} & \cdots & a_{2, i_{k}} \beta^{i_{k}-1} \\
\vdots & \vdots & \ddots & \vdots \\
a_{k, i_{1}} \beta^{i_{1}-k+1} & a_{k, i_{2}} \beta^{i_{2}-k+1} & \cdots & a_{k, i_{k}} \beta^{i_{k}-k+1}
\end{array}\right)
$$

where $\left\{i_{1}, i_{2}, \ldots, i_{k}\right\} \subseteq\{k, k+1, \ldots, n-2\}$, such that the degree of $\operatorname{det}\left(\mathbf{M}_{1}\right)$ is the same as that of $\operatorname{det}\left(\mathbf{D}_{k}\right)$, and their leading coefficients are the same. Then

$$
\begin{aligned}
& \operatorname{det}\left(\mathbf{M}_{1}\right)=\operatorname{det}\left(\begin{array}{cccc}
a_{1, i_{1}} \beta^{k-1} & a_{1, i_{2}} \beta^{k-1} & \cdots & a_{1, i_{k}} \beta^{k-1} \\
a_{2, i_{1}} \beta^{k-2} & a_{2, i_{2}} \beta^{k-2} & \cdots & a_{2, i_{k}} \beta^{k-2} \\
\vdots & \vdots & \ddots & \vdots \\
a_{k, i_{1}} & a_{k, i_{2}} & \cdots & a_{k, i_{k}}
\end{array}\right) \cdot\left(\beta^{i_{1}-k+1} \beta^{i_{2}-k+1} \cdots \beta^{i_{k}-k+1}\right)= \\
& \operatorname{det}\left(\begin{array}{cccc}
a_{1, i_{1}} & a_{1, i_{2}} & \cdots & a_{1, i_{k}} \\
a_{2, i_{1}} & a_{2, i_{2}} & \cdots & a_{2, i_{k}} \\
\vdots & \vdots & \ddots & \vdots \\
a_{k, i_{1}} & a_{k, i_{2}} & \cdots & a_{k, i_{k}}
\end{array}\right) \cdot\left(\beta^{k-1} \beta^{k-2} \cdots \beta\right) \cdot\left(\beta^{i_{1}-k+1} \beta^{i_{2}-k+1} \cdots \beta^{i_{k}-k+1}\right),
\end{aligned}
$$

whose degree is $k(k-1) / 2+\sum_{j=1}^{k}\left(i_{j}-k+1\right) \leq k n-k^{2}-k<m$. Since every $k$-minor of $\mathbf{A}_{1}$ is nonzero, the leading coefficient of $\operatorname{det}\left(\mathbf{M}_{1}\right)$ is nonzero. So $\operatorname{det}\left(\mathbf{D}_{k}\right) \neq 0$.

Subcase 1.2. $\mathbf{D}_{k}$ contains $h$ columns coming from the first $k$ columns of GB for some $1 \leq h \leq k$. Write these $h$ columns as the $j_{1}$-th, $j_{2}$-th, $\ldots, j_{h}$-th columns. Let $\mathbf{U}_{k \times h}$ be the submatrix formed by the first $h$ columns of $\mathbf{D}_{k}$. Take

$$
\mathbf{M}_{2}=\left(\begin{array}{c:cccc}
\mathbf{U}_{k \times h} & a_{1, i_{h+1}} \beta^{i_{h+1}} & a_{1, i_{h+2}} \beta^{i_{h+2}} & \cdots & a_{1, i_{k}} \beta^{i_{k}} \\
& a_{2, i_{h+1}} \beta^{i_{h+1}-1} & a_{2, i_{h+2}} \beta^{i_{h+2}-1} & \cdots & a_{2, i_{k}} \beta^{i_{k}-1} \\
& \vdots & \vdots & \ddots & \vdots \\
& a_{k, i_{h+1}} \beta^{i_{h+1}-k+1} & a_{k, i_{h+2}} \beta^{i_{h+2}-k+1} & \cdots & a_{k, i_{k}} \beta^{i_{k}-k+1}
\end{array}\right),
$$

where $\left\{i_{h+1}, i_{h+2}, \ldots, i_{k}\right\} \subseteq\{k, k+1, \ldots, n-2\}$, such that the degree of $\operatorname{det}\left(\mathbf{M}_{2}\right)$ is the same as that of $\operatorname{det}\left(\mathbf{D}_{k}\right)$, and their leading coefficients are the same. Then

$$
\operatorname{det}\left(\mathbf{M}_{2}\right)=\operatorname{det}\left(\begin{array}{c:cccc} 
& a_{1, i_{h+1}} \beta^{k-1} & a_{1, i_{h+2}} \beta^{k-1} & \cdots & a_{1, i_{k}} \beta^{k-1} \\
\mathbf{U}_{k \times h} & a_{2, i_{h+1} \beta^{k-2}} & a_{2, i_{h+2}} \beta^{k-2} & \cdots & a_{2, i_{k} \beta^{k-2}} \\
& \vdots & \vdots & \ddots & \vdots \\
& a_{k, i_{h+1}} & a_{k, i_{h+2}} & \cdots & a_{k, i_{k}}
\end{array}\right) \cdot\left(\beta^{i_{h+1}-k+1} \beta^{i_{h+2}-k+1} \ldots \beta^{i_{k}-k+1}\right) .
$$

Clearly, compared with the degree of $\operatorname{det}\left(\mathbf{M}_{1}\right)$, the $\operatorname{degree}$ of $\operatorname{det}\left(\mathbf{M}_{2}\right)$ is less than $m$. Let $\mathbf{L}$ be a $(k-h) \times(k-h)$ matrix obtained by removing the $j_{1}$-th, $j_{2}$-th, $\ldots, j_{h}$-th rows from the following matrix

$$
\left(\begin{array}{ccc}
a_{1, i_{h+1}} & \cdots & a_{1, i_{k}} \\
\vdots & \ddots & \vdots \\
a_{k, i_{h+1}} & \cdots & a_{k, i_{k}}
\end{array}\right)
$$

It is readily checked that the leading coefficient of $\operatorname{det}\left(\mathbf{M}_{2}\right)$ is $\operatorname{det}(\mathbf{L})$ or $-\operatorname{det}(\mathbf{L})$ (this fact comes from two observations: (1) via elementary row-addition operations on $\operatorname{det}\left(\mathbf{M}_{2}\right)$, the $\mathbf{U}_{k \times h}$ part in $\mathbf{M}_{2}$, which is an upper triangular matrix, can be transformed to a matrix with at most one 1 in each row; (2) $\beta$ has higher degrees in upper rows of $\mathbf{M}_{2}$ ). Since $\mathbf{L}$ is a minor of $\mathbf{A}_{1}, \operatorname{det}(\mathbf{L}) \neq 0$. So $\operatorname{det}\left(\mathbf{D}_{k}\right) \neq 0$.

Case 2. $\mathbf{D}_{k}$ contains the last column of $\mathbf{G B}$. The arguments are similar to those in Case 1.

Subcase 2.1. $\mathbf{D}_{k}$ doesn't contain any of the first $k$ columns of GB. Take 


$$
\mathbf{M}_{3}=\left(\begin{array}{ccccc}
a_{1, i_{1}} \beta^{i_{1}} & a_{1, i_{2}} \beta^{i_{2}} & \cdots & a_{1, i_{k-1}} \beta^{i_{k-1}} & a_{1, n-1} \beta^{n} \\
a_{2, i_{1}} \beta^{i_{1}-1} & a_{1, i_{2}} \beta^{i_{2}-1} & \cdots & a_{2, i_{k-1}} \beta^{i_{k-1}-1} & a_{2, n-1} \beta^{n-2} \\
\vdots & \vdots & \ddots & \vdots & \vdots \\
a_{k, i_{1}} \beta^{i_{1}-k+1} & a_{k, i_{2}} \beta^{i_{2}-k+1} & \cdots & a_{k, i_{k-1}} \beta^{i_{k-1}-k+1} & a_{k, n-1} \beta^{n-k}
\end{array}\right)
$$

where $\left\{i_{1}, i_{2}, \ldots, i_{k-1}\right\} \subseteq\{k, k+1, \ldots, n-2\}$, such that the $\operatorname{degree}$ of $\operatorname{det}\left(\mathbf{M}_{3}\right)$ is the same as that of $\operatorname{det}\left(\mathbf{D}_{k}\right)$, and their leading coefficients are the same. Then

$$
\begin{aligned}
& \operatorname{det}\left(\mathbf{M}_{3}\right)=\operatorname{det}\left(\begin{array}{ccccc}
a_{1, i_{1}} \beta^{k-1} & a_{1, i_{2}} \beta^{k-1} & \ldots & a_{1, i_{k-1}} \beta^{k-1} & a_{1, n-1} \beta^{k} \\
a_{2, i_{1}} \beta^{k-2} & a_{2, i_{2}} \beta^{k-2} & \cdots & a_{2, i_{k-1}} \beta^{k-2} & a_{2, n-1} \beta^{k-2} \\
\vdots & \vdots & \ddots & \vdots & \vdots \\
a_{k, i_{1}} & a_{k, i_{2}} & \cdots & a_{k, i_{k-1}} & a_{k, n-1}
\end{array}\right) \cdot\left(\beta^{i_{1}-k+1} \ldots \beta^{i_{k-1}-k+1} \beta^{n-k}\right) \\
& =\operatorname{det}\left(\begin{array}{ccccc}
a_{1, i_{1}} & a_{1, i_{2}} & \cdots & a_{1, i_{k-1}} & a_{1, n-1} \beta \\
a_{2, i_{1}} & a_{2, i_{2}} & \cdots & a_{2, i_{k-1}} & a_{2, n-1} \\
\vdots & \vdots & \ddots & \vdots & \\
a_{k, i_{1}} & a_{k, i_{2}} & \cdots & a_{k, i_{k-1}} & a_{k, n-1}
\end{array}\right) \cdot\left(\beta^{k-1} \beta^{k-2} \cdots \beta\right) \cdot\left(\beta^{i_{1}-k+1} \cdots \beta^{i_{k-1}-k+1} \beta^{n-k}\right),
\end{aligned}
$$

whose degree is $1+k(k-1) / 2+\sum_{j=1}^{k-1}\left(i_{j}-k+1\right)+n-k \leq k n-k^{2}+1<m$. Since $a_{1, n-1} \in \mathbb{F}_{q}^{*}$ and every $(k-1)$-minor of $\mathbf{A}_{1}$ is nonzero, the leading coefficient of $\operatorname{det}\left(\mathbf{M}_{3}\right)$ is nonzero. So $\operatorname{det}\left(\mathbf{D}_{k}\right) \neq 0$.

Subcase 2.2. $\mathbf{D}_{k}$ contains $h$ columns coming from the first $k$ columns of GB for some $1 \leq h \leq k$. Write these $h$ columns as the $j_{1}$-th, $j_{2}$-th, $\ldots, j_{h}$-th columns. Let $\mathbf{U}_{k \times h}$ be the submatrix formed by the first $h$ column of $\mathbf{D}_{k}$. Take

$$
\mathbf{M}_{4}=\left(\begin{array}{c:cccc} 
& a_{1, i_{h+1}} \beta^{i_{h+1}} & \cdots & a_{1, i_{k-1}} \beta^{i_{k-1}} & a_{1, n-1} \beta^{n} \\
\mathbf{U}_{k \times h} & a_{2, i_{h+1}} \beta^{i_{h+1}-1} & \cdots & a_{2, i_{k-1}} \beta^{i_{k-1}-1} & a_{2, n-1} \beta^{n-2} \\
& \vdots & \ddots & \vdots & \\
& a_{k, i_{h+1}} \beta^{i_{h+1}-k+1} & \cdots & a_{k, i_{k-1}} \beta^{i_{k-1}-k+1} & a_{k, n-1} \beta^{n-k}
\end{array}\right)
$$

where $\left\{i_{h+1}, i_{h+2}, \ldots, i_{k-1}\right\} \subseteq\{k, k+1, \ldots, n-2\}$, such that the degree of $\operatorname{det}\left(\mathbf{M}_{4}\right)$ is the same as that of $\operatorname{det}\left(\mathbf{D}_{k}\right)$, and their leading coefficients are the same. Then

$\operatorname{det}\left(\mathbf{M}_{4}\right)=\operatorname{det}\left(\begin{array}{c:cccc} & a_{1, i_{h+1}} \beta^{k-1} & \cdots & a_{1, i_{k-1}} \beta^{k-1} & a_{1, n-1} \beta^{k} \\ & a_{2, i_{h+1}} \beta^{k-2} & \cdots & a_{2, i_{k-1}} \beta^{k-2} & a_{2, n-1} \beta^{k-2} \\ & \vdots & \ddots & \vdots & \vdots \\ & a_{k, i_{h+1}} & \cdots & a_{k, i_{k-1}} & a_{k, n-1}\end{array}\right) \cdot\left(\beta^{i_{h+1}-k+1} \cdots \beta^{i_{k-1}-k+1} \beta^{n-k}\right)$,

Clearly, compared with the degree of $\operatorname{det}\left(\mathbf{M}_{3}\right)$, the degree of $\operatorname{det}\left(\mathbf{M}_{4}\right)$ is less than $m$.

Subcase 2.2.1. $\mathbf{D}_{k}$ contains the first column of GB. W.l.o.g., assume that the $j_{1}$-th column of GB is just its first column. Let $\mathbf{L}$ be a $(k-h) \times(k-h)$ matrix obtained by removing the $j_{1}$-th, $j_{2}$-th, $\ldots, j_{h}$-th rows from the following matrix

$$
\left(\begin{array}{cccc}
a_{1, i_{h+1}} & \cdots & a_{1, i_{k-1}} & a_{1, n-1} \\
\vdots & \ddots & \vdots & \vdots \\
a_{k, i_{h+1}} & \cdots & a_{k, i_{k-1}} & a_{k, n-1}
\end{array}\right) .
$$

It is readily checked that the leading coefficient of $\operatorname{det}\left(\mathbf{M}_{4}\right)$ is $\operatorname{det}(\mathbf{L})$ or $-\operatorname{det}(\mathbf{L})$. Since $\mathbf{L}$ is a minor of $\mathbf{A}_{2}, \operatorname{det}(\mathbf{L}) \neq 0$. So $\operatorname{det}\left(\mathbf{D}_{k}\right) \neq 0$.

Subcase 2.2.2. $\mathbf{D}_{k}$ does not contain the first column of GB. Let $\mathbf{L}$ be a $(k-h-$ $1) \times(k-h-1)$ matrix obtained by removing the first, the $j_{1}$-th, $j_{2}$-th, $\ldots, j_{h}$-th rows from the following matrix 


$$
\left(\begin{array}{cccc}
a_{1, i_{h+1}} & \cdots & a_{1, i_{k-1}} & a_{1, n-1} \\
\vdots & \ddots & \vdots & \vdots \\
a_{k, i_{h+1}} & \cdots & a_{k, i_{k-1}} & a_{k, n-1}
\end{array}\right) .
$$

It is readily checked that the leading coefficient of $\operatorname{det}\left(\mathbf{M}_{4}\right)$ is $a_{1, n-1} \cdot \operatorname{det}(\mathbf{L})$ or $-a_{1, n-1}$. $\operatorname{det}(\mathbf{L})$. Note that $a_{1, n-1} \in \mathbb{F}_{q}^{*}$. Since $\mathbf{L}$ is a minor of $\mathbf{A}_{2}, \operatorname{det}(\mathbf{L}) \neq 0$. So $\operatorname{det}\left(\mathbf{D}_{k}\right) \neq 0$.

\section{B Appendix}

Proof of Lemma 3.11 To construct the required matrix $\mathbf{G}$, we first take a $\mathcal{G}[\mu \times(\eta-$ $r), d]_{q}$ code in vector representation over $\mathbb{F}_{q^{\mu}}$ :

$$
\mathbf{G}_{0}=\left(\begin{array}{cccc}
1 & g_{0,1} & \cdots & g_{0, \eta-r-1} \\
1 & g_{0,1}^{[1]} & \cdots & g_{0, \eta-r-1}^{[1]} \\
\vdots & \vdots & \ddots & \vdots \\
1 & g_{0,1}^{[\kappa-1]} & \cdots & g_{0, \eta-r-1}^{[\kappa-1]}
\end{array}\right),
$$

where $1, g_{0,1}, \ldots, g_{0, \eta-r-1} \in \mathbb{F}_{q^{\mu}}$ are linearly independent over $\mathbb{F}_{q}$.

We shall extend $\mathbf{G}_{0}$ by adding $r$ columns to obtain $\mathbf{G}$. We need $r$ steps. For $0 \leq i \leq$ $r-1$, in Step $i$, let $\omega_{i}=\eta-r+i-2$ and $\mathbf{G}_{i}=$

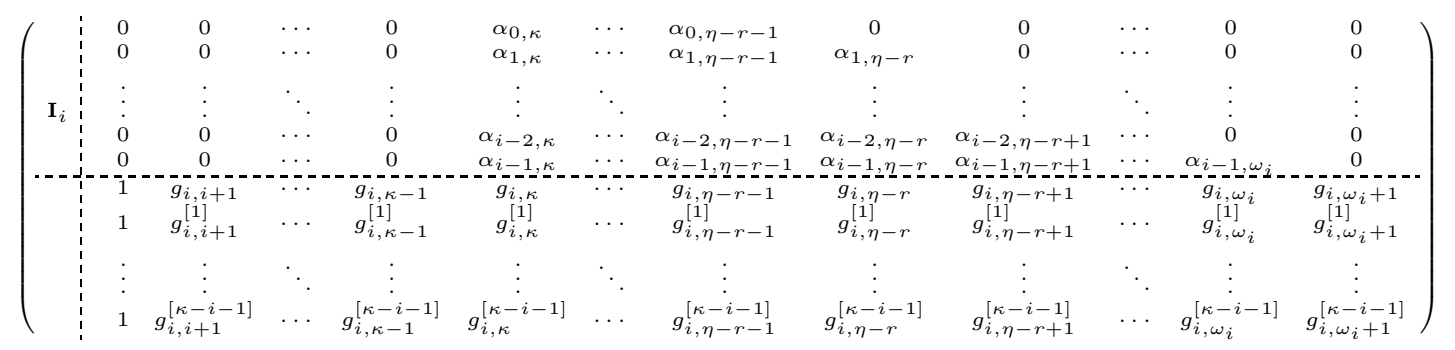

be a $\kappa \times\left(\omega_{i}+2\right)$ matrix, where $1, g_{i, i+1}, \ldots, g_{i, \omega_{i}+1} \in \mathbb{F}_{q^{\mu}}$ are linearly independent over $\mathbb{F}_{q}$, and the sub-matrix of $\mathbf{G}_{i}$ obtained by removing its first $i$ rows and the leftmost $i$ columns produces a $\mathcal{G}[\mu \times(\eta-r), d+i]_{q}$ code. When $i=0, \mathbf{G}_{i}$ is just $\mathbf{G}_{0}$ we defined in the above paragraph. Now, we show that how to obtain $\mathbf{G}_{i+1}$ from $\mathbf{G}_{i}$ for $0 \leq i \leq r-1$.

Let $t_{i, i+1}, t_{i, i+2}, \ldots, t_{i, \kappa-1} \in \mathbb{F}_{q^{\mu}}$ such that

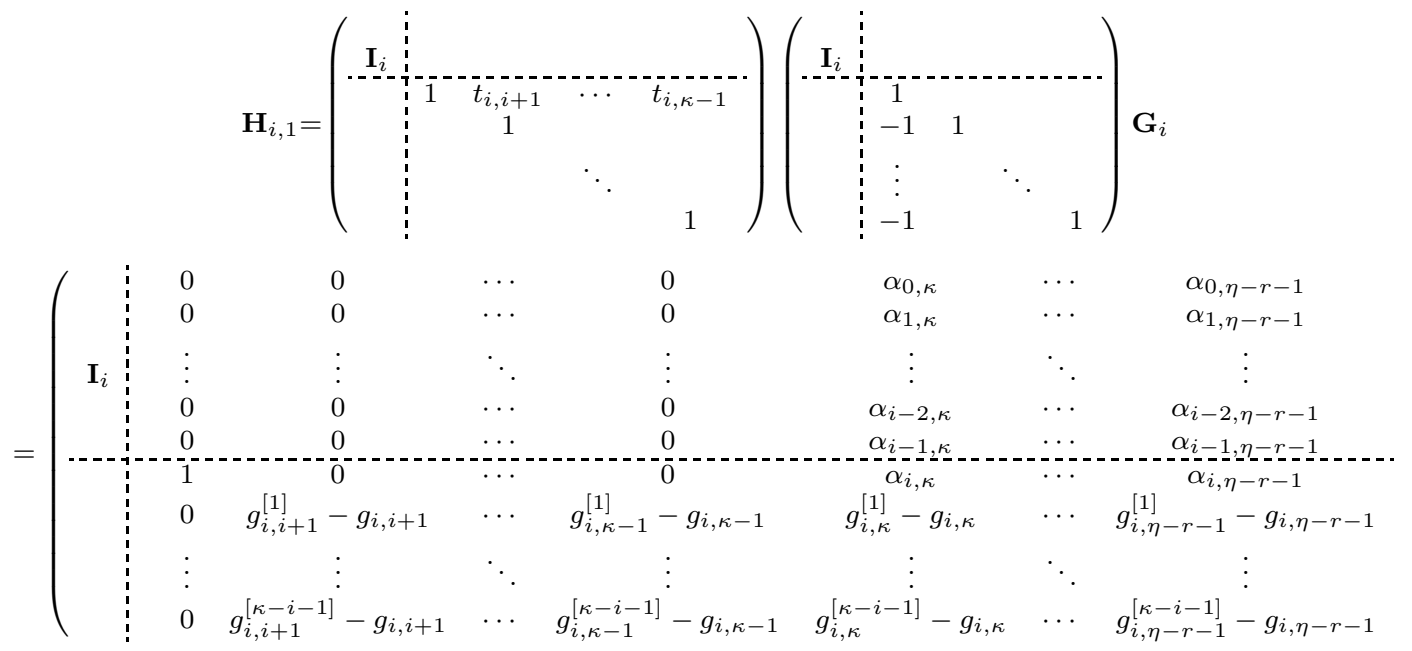




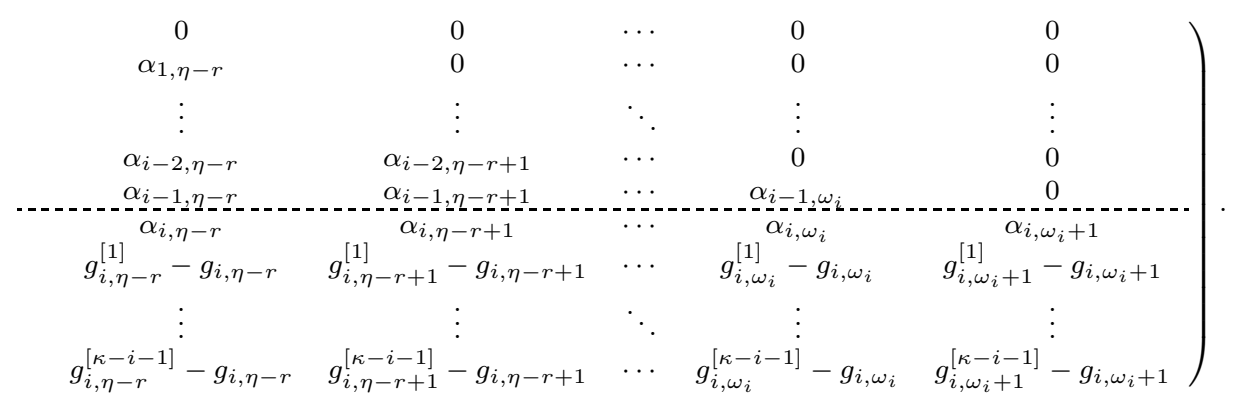

Notice that $t_{i, i+1}, \ldots, t_{i, \kappa-1}$ influence only the first row under the broken line of $\mathbf{H}_{i, 1}$ and the requirements on this row constitute a linear system of equations with $\kappa-1-i$ equations and $\kappa-1-i$ unknowns. Therefore, the desired $t_{i, i+1}, \ldots, t_{i, \kappa-1}$ always exist (this is from the observation of the generator matrix of the Gabidulin code defined by $\left.1, g_{i, i+1}, \ldots, g_{i, \kappa-1}\right)$.

Let

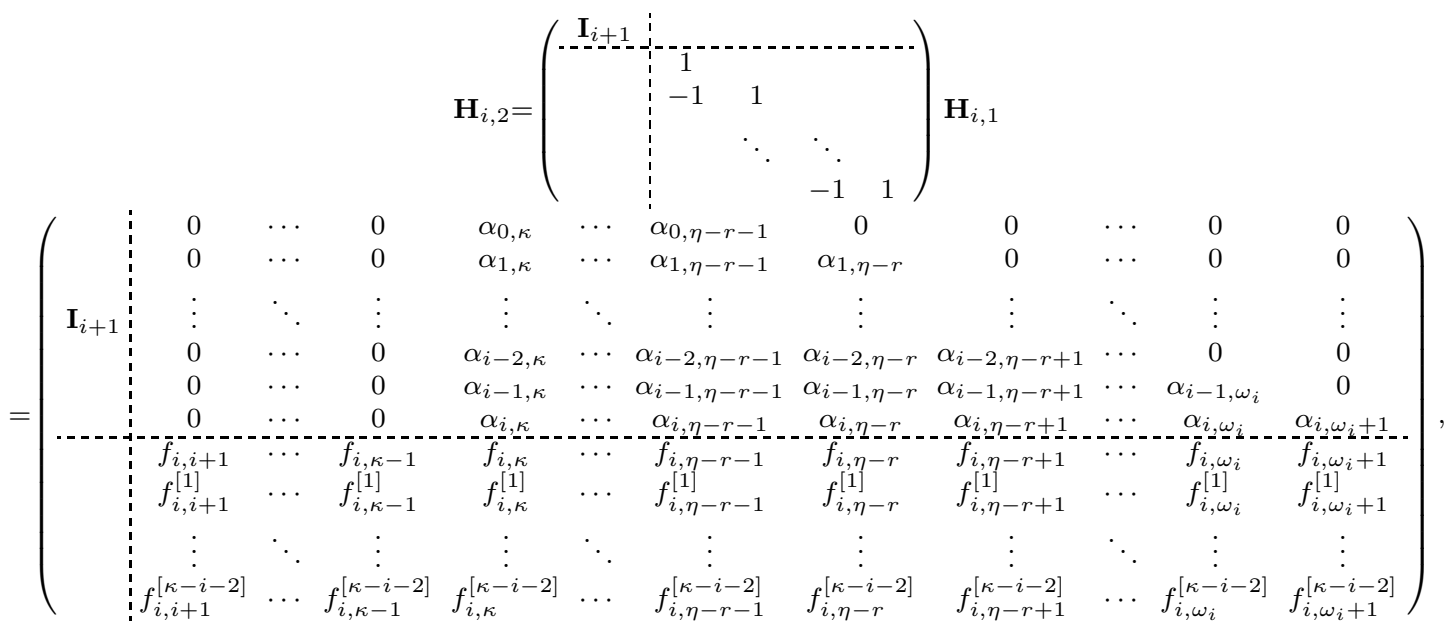

where $f_{i, j}=g_{i, j}^{[1]}-g_{i, j}$ for $i+1 \leq j \leq \omega_{i}+1$. For any full-rank matrix $\mathbf{T}_{i} \in \mathbb{F}_{q^{\mu}}^{\kappa \times \kappa}$, the generator matrix $\mathbf{T}_{i} \mathbf{G}_{i}$ defines the same code as $\mathbf{G}_{i}$, so $\mathbf{H}_{i, 2}$ defines the same code as $\mathbf{G}_{i}$.

We can assert that $f_{i, i+1}, f_{i, i+2}, \ldots, f_{i, \omega_{i}+1} \in \mathbb{F}_{q^{\mu}}$ are linearly independent over $\mathbb{F}_{q}$. Since $1, g_{i, i+1}, \ldots, g_{i, \omega_{i}+1} \in \mathbb{F}_{q^{\mu}}$ are linearly independent over $\mathbb{F}_{q}$, we construct a $\mathcal{G}[\mu \times$ $\left.\left(\omega_{i}-i+2\right), \omega_{i}-i+1\right]_{q}$ code generated by

$$
\left(\begin{array}{cccc}
1 & g_{i, i+1} & \cdots & g_{i, \omega_{i}+1} \\
1 & g_{i, i+1}^{[1]} & \cdots & g_{i, \omega_{i}+1}^{[1]}
\end{array}\right) .
$$

Since $\left(0, f_{i, i+1}, \ldots, f_{i, \omega_{i}+1}\right)$ is a codeword of the $\mathcal{G}\left[\mu \times\left(\omega_{i}-i+2\right), \omega_{i}-i+1\right]_{q}$ code, then $\operatorname{rank}\left(f_{i, i+1}, \ldots, f_{i, \omega_{i}+1}\right)=\omega_{i}-i+1$. So, $f_{i, i+1}, f_{i, i+2}, \ldots, f_{i, \omega_{i}+1}$ are linearly independent over $\mathbb{F}_{q}$.

Additionally, since $\mu \geq \eta-r=\omega_{i}-i+2$, there exists an element $f_{i, \omega_{i}+2} \in \mathbb{F}_{q^{\mu}}$ which is $\mathbb{F}_{q}$-linearly independent of $f_{i, i+1}, \ldots, f_{i, \omega_{i}+1}$. Hence, the $\kappa \times\left(\omega_{i}+3\right)$ matrix

$$
\mathbf{H}_{i, 3}=\left(\begin{array}{c:c} 
& \mathbf{0}_{(i+1) \times 1} \\
& f_{i, \omega_{i}+2} \\
\mathbf{H}_{i, 2} & f_{i, \omega_{i}+2}^{[1]} \\
& \vdots \\
& f_{i, \omega_{i}+2}^{[\kappa-i-2]}
\end{array}\right)
$$


defines with its right bottom $(\kappa-i-1) \times\left(\omega_{i}-i+2\right)$ submatrix a $\mathcal{G}\left[\mu \times\left(\omega_{i}-i+2\right), d+i+1\right]_{q}$ code.

Now we set

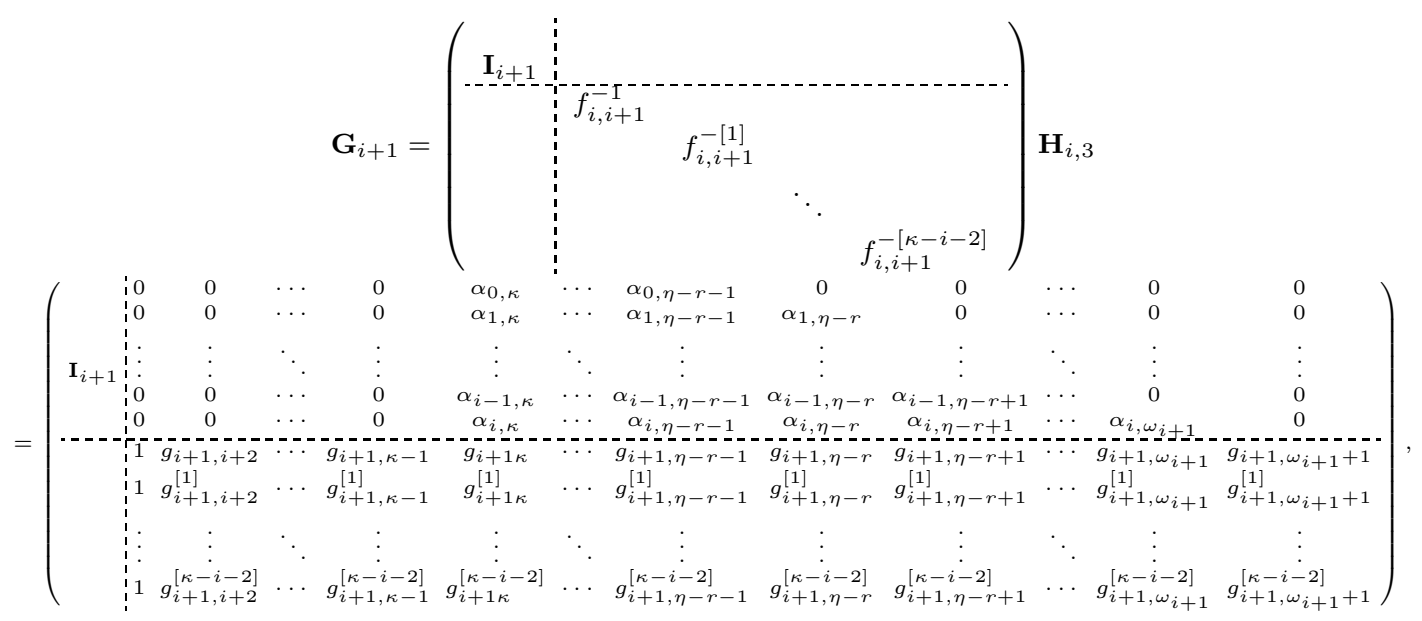

where $\omega_{i+1}=\omega_{i}+1$ and $g_{i+1, j}=f_{i, j} f_{i, i+1}^{-1}$ for $j \in\left\{i+2, \ldots, \omega_{i+1}+1\right\}$. Notice that $1, g_{i+1, i+2}, \ldots, g_{i+1, \omega_{i+1}+1}$ are linearly independent over $\mathbb{F}_{q}$, and the right bottom $(\kappa-i-$ $1) \times\left(\omega_{i+1}-i+1\right)$ submatrix of $\mathbf{G}_{i+1}$ can produce the same $\mathcal{G}\left[\mu \times\left(\omega_{i}-i+2\right), d+i+1\right]_{q}$ code as the one produced by $\mathbf{H}_{i, 3}$.

Finally, we can choose an invertible matrix $\mathbf{T} \in \mathbb{F}_{q^{\mu}}^{(\kappa-r) \times(\kappa-r)}$ such that

$$
\mathbf{G}=\left(\begin{array}{cc}
\mathbf{I}_{r \times r} & \\
& \mathbf{T}
\end{array}\right) \cdot \mathbf{G}_{r}
$$

is our required matrix.

\section{Acknowledgements}

The authors thank the anonymous referees for their valuable comments and suggestions that helped improve the equality of the paper.

\section{References}

[1] J. Antrobus and H. Gluesing-Luerssen, Maximal Ferrers diagram codes: constructions and genericity considerations, arXiv:1804.00624v1.

[2] R. Ahlswede, N. Cai, S.-Y.R. Li, and R.W. Yeung, Network information flow, IEEE Trans. Inf. Theory, 46 (2000), 1204-1216.

[3] E. Ballico, Linear subspaces of matrices associated to a Ferrers diagram and with a prescribed lower bound for their rank, Linear Algebra and its Appl., 483 (2015), 30-39.

[4] P. Delsarte, Bilinear forms over a finite field, with applications to coding theory, $J$. Combin. Theory A, 25 (1978), 226-241.

[5] T. Etzion, E. Gorla, A. Ravagnani and A. Wachter-Zeh, Optimal Ferrers diagram rank-metric codes, IEEE Trans. Inf. Theory, 62 (2016), 1616-1630. 
[6] T. Etzion and N. Silberstein, Error-correcting codes in projective spaces via rankmetric codes and Ferrers diagrams, IEEE Trans. Inf. Theory, 55 (2009), 2909-2919.

[7] T. Etzion and N. Silberstein, Codes and designs related to lifted MRD codes, IEEE Trans. Inf. Theory, 59 (2013), 1004-1017.

[8] T. Etzion and A. Vardy, Error-correcting codes in projective spaces, IEEE Trans. Inf. Theory, 57 (2011), 1165-1173.

[9] È.M. Gabidulin, Theory of codes with maximum rank distance, Problems Inf. Transmiss., 21 (1985), 3-16.

[10] M. Gadouleau and Z. Yan, Constant-rank codes and their connection to constantdimension codes, IEEE Trans. Inform. Theory, 56 (2010), 3207-3216.

[11] E. Gorla and A. Ravagnani, Subspace codes from Ferrers diagrams, J. Algebra and its Appl., 16 (2017), 1750131.

[12] R. Kötter and F.R. Kschischang, Coding for errors and erasures in random network coding, IEEE Trans. Inf. Theory, 54 (2008), 3579-3591.

[13] A. Kohnert and S. Kurz, Construction of large constant dimension codes with a prescribed minimum distance, Lecture Notes Comp. Sci., 5393 (2008), 31-42.

[14] F.J. MacWilliams and N.J.A. Sloane, The Theory of Error-Correcting Codes, Amsterdam, The Netherlands: North Holland, 1988.

[15] R.M. Roth, Maximum-rank array codes and their application to crisscross error correction, IEEE Trans. Inf. Theory, 37 (1991), 328-336.

[16] J. Sheekey, A new family of linear maximum rank distance codes, Adv. Math. Commun., 10 (2016), 475-488.

[17] N. Silberstein and T. Etzion, Large constant dimension codes and lexicodes, Adv. in Math. of Comm., 5 (2011), 177-189.

[18] N. Silberstein and T. Etzion, Enumerative coding for Grassmannian space, IEEE Trans. Inf. Theory, 57 (2011), 365-374.

[19] N. Silberstein and A.-L. Trautmann, Subspace codes based on graph matchings, Ferrers diagrams, and pending blocks, IEEE Trans. Inf. Theory, 61 (2015), 39373953.

[20] D. Silva, F.R. Kschischang, and R. Kötter, A rank-metric approach to error control in random network coding, IEEE Trans. Inf. Theory, 54 (2008), 3951-3967.

[21] V. Skachek, Recursive code construction for random networks, IEEE Trans. Inf. Theory, 56 (2010), 1378-1382.

[22] A.-L. Trautmann, F. Manganiello, M. Braun and J. Rosenthal, Cyclic orbit codes, IEEE Trans. Inf. Theory, 59 (2013), 7386-7404.

[23] A.-L. Trautmann and K. Marshall, New criteria for MRD and Gabidulin codes and some rank-metric code constructions, Advances in Mathematics of Communications, 11 (2017), 533-548. 
[24] A.-L. Trautmann and J. Rosenthal, New improvements on the Echelon-Ferrers construction, in Proc. 19th Int. Symp. Math. Theory Netw. Syst., Jul. 2010, 405-408.

[25] T. Zhang and G. Ge, Constructions of optimal Ferrers diagram rank metric codes, Des. Codes Cryptogr., doi:10.1007/s10623-018-0491-4. 